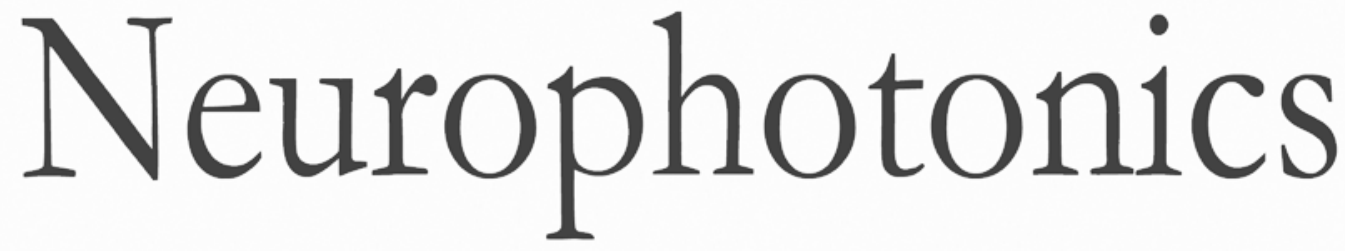

\title{
Longitudinal study of hemodynamics and dendritic membrane potential changes in the mouse cortex following a soft cranial window installation
}

Hyejin Park Nayeon You Juheon Lee Minah Suh 


\title{
Longitudinal study of hemodynamics and dendritic membrane potential changes in the mouse cortex following a soft cranial window installation
}

\author{
Hyejin Park, ${ }^{a, b, c}$ Nayeon You, ${ }^{a, d}$ Juheon Lee, ${ }^{a, d}$ and Minah Suh ${ }^{a, c, d, e, *}$ \\ ${ }^{a}$ Institute for Basic Science, Center for Neuroscience Imaging Research, Suwon, Republic of Korea \\ bSungkyunkwan University, Department of Biological Sciences, Suwon, Republic of Korea \\ 'Sungkyunkwan University, Biomedical Institute for Convergence, Suwon, Republic of Korea \\ dSungkyunkwan University, Department of Biomedical Engineering, Suwon, Republic of Korea \\ 'Sungkyunkwan University, SAHIST, Suwon, Republic of Korea
}

\begin{abstract}
The soft cranial window using polydimethylsiloxane allows direct multiple access to neural tissue during long-term monitoring. However, the chronic effects of soft window installation on the brain have not been fully studied. Here, we investigate the long-term effects of soft window installation on sensory-evoked cerebral hemodynamics and neuronal activity. We monitored the brain tissue immunocytohistology for 6 weeks postinstallation. Heightened reactive astrocytic and microglia levels were found at 2 weeks postinstallation. By 6 weeks postinstallation, mice had expression levels similar to those of normal animals. We recorded sensory-evoked hemodynamics of the barrel cortex and LFP during whisker stimulation at these time points. Animals at 6 weeks postinstallation showed stronger hemodynamic responses and focalized barrel mapping than 2-week postoperative mice. LFP recordings of 6-week postoperative mice also showed higher neural activity at the barrel column corresponding to the stimulated whisker. Furthermore, the expression level of interleukin- $1 \beta$ was highly upregulated at 2 weeks postinstallation. When we treated animals postoperatively with minocycline plus $\mathrm{N}$-acetylcystein, a drug-suppressing inflammatory cytokine, these animals did not show declined hemodynamic responses and neuronal activities. This result suggests that neuroinflammation following soft window installation may alter hemodynamic and neuronal responses upon sensory stimulation. ๑ The Authors. Published by SPIE under a Creative Commons Attribution 4.0 Unported License. Distribution or reproduction of this work in whole or in part requires full attribution of the original publication, including its DOI. [DOI: 10.1117/1.NPh.6.1.015006]
\end{abstract}

Keywords: cranial window; neurovascular coupling; intrinsic optical signal; sensory stimulation; polydimethylsiloxane; craniotomy.

Paper 18061RR received Oct. 10, 2018; accepted for publication Jan. 25, 2019; published online Feb. 25, 2019.

\section{Introduction}

Optic-based in vivo imaging has recently become a popular technique to study living brain function, owing to technical advances including two-photon microscopy, fluorescent dye development, and genetic modulation to express fluorescence. In addition, the superb spatial and temporal resolution of optic-based imaging as compared to other imaging methodologies (e.g., MRI, PET) has led many neuroscientists to adopt optic-based techniques. However, due to the nature of light, the skull is an impenetrable obstacle in in vivo optical imaging; thus, the skull needs to be removed or modified for better imaging. These types of procedures obviously affect the physiological environment of the brain. In turn, these environmental changes can influence basic brain functions, such as neurovascular coupling (NVC). ${ }^{1} \mathrm{NVC}$ is a cascade of events among neurons, glias, and the vascular system that regulates the cerebral metabolic demands associated with neuronal activation. At this time, little is known about the influence of cranial window installation on NVC over time. Understanding the exact temporal changes of cerebral hemodynamics and neuronal activation associated with the cranial window installation process will be beneficial for neuroscientists.

Several cranial window systems are used for optical imaging: common glass-top, ${ }^{2}$ thin-skull, ${ }^{3}$ and reinforced thin-skull. ${ }^{4,5}$

*Address all correspondence to Minah Suh, E-mail: minahsuh@skku.edu
Growing interest in combining imaging with other research modalities, such as electrophysiological recording and drug or chemical injection, creates the need to develop a cranial window system. In particular, incorporating a hole within a glasstop window ${ }^{6}$ or using a soft cranial window installation ${ }^{7}$ add ways to penetrate the cranial window with needles and electrodes during imaging. The glass-top window is a widely used methodology for in vivo imaging, and some groups are currently actively modifying the glass-top window to facilitate electrophysiological recording or chemical injection. ${ }^{8}$ Alternatively, a soft cranial window system utilizes transparent, elastic, and biocompatible silicone-based polydimethylsiloxane (PDMS) as a coverslip. ${ }^{7}$ This relatively simple window allows not only long-term chronic in vivo imaging but also multiple penetrations across the entire window, allowing electrophysiological recording and drug intervention. However, one inevitable surgical step for soft cranial window systems is the removal of the skull or both the skull and dura.

Even drilling the skull with some pressure can cause acute brain injury, inducing vascular damage and meningeal cell death. ${ }^{9}$ Therefore, a full craniotomy-duratomy brings a certain degree of alteration to the tissue environment and physiology no matter how careful the surgery. As compared to thin-skull surgery, open-skull surgery activated microglia and extensive glial fibrillary acidic protein (GFAP) expression at the surgical site. ${ }^{4,10}$ Glial activation persisted for at least 4 weeks and was highly associated with neuronal spine turnover. Repeated in vivo 
two-photon microscopic imaging showed that the astrocyte number significantly increased following open-skull surgery and that the astrocyte size began to decrease 3 days postsurgery. ${ }^{11}$ This astrogliosis was sustained for 4 to 7 weeks. Open-skull surgery also creates vasculature changes, as revealed by Texas red-dextran labeled vasculature. ${ }^{4}$ Using a chronic cranial window model, focusing on the surface vasculature in particular, veins exhibited significantly altered topological properties when they were compared at 2 days and 3 weeks after the installation. Other cortical vasculature alterations following open-skull surgery include large vasodilation at 3 days postsurgery. In fact, some vessels remain in a dilated state even 56 days after the craniotomy. ${ }^{12}$

Despite this neurobiological information in relation to open-skull chronic cranial window implantation, no systematic studies concerning functional alterations following open-skull surgery have been performed. In particular, we barely know how a full craniotomy and PDMS material affect cerebral hemodynamics and neuronal activation in relation to normal sensory processing. PDMS is known as a biocompatible material, and it is highly utilized as an implant substance in the biomedical field, but the long-term implantation of PDMS into the brain of mice for functional imaging has barely been utilized until now. In addition, its properties, such as hydrophobicity and flexibility, are quite different from those of glass, which is commonly used as a coverslip of open-skull cranial windows in this field.

Since NVC is an important marker in dysfunctional brains, ${ }^{13}$ a deeper understanding of the long-term effects of soft cranial window implantation on both cerebral hemodynamics and dendritic membrane potential is critically important. In this study, we investigated how soft cranial window installation, which we previously demonstrated its feasibility for chronic brain study, affects postsurgical and whisker-stimulated cerebral hemodynamics and neuronal activation.

\section{Materials and Methods}

\subsection{Animal Preparation}

Male C57BL/6 mice weighing 22 to $28 \mathrm{~g}$ were used for this study. In total, 90 mice were used, and 10 of those mice did not undergo craniotomy procedures, serving as the control group. All animal procedures were performed under guidelines set by the Institutional Animal Care and Use Committee of Sungkyunkwan University.

\subsection{Cranial Window Installation}

Animals were anesthetized by inhalation with isoflurane (3\% for the induction of anesthesia and $1.5 \%$ for maintenance during the surgical procedure; Hana Pharm, Republic of Korea). They were then fixed in a stereotaxic frame (David Kopf Instruments) for a full craniotomy with their body temperature kept at $37^{\circ} \mathrm{C}$ to $37.5^{\circ} \mathrm{C}$ using a heating pad-connected temperature controller (DC temperature controller, FHC). The skin on the mouse's head was sanitized with an alcohol swab (isopropyl alcohol $70 \%, \mathrm{BD})$ and cut with surgical scissors. Then, the epidermal skin was removed, and cyanoacrylate glue (Loctite) was applied gently to hold the residual skin.

To monitor brain waves of the entire brain, a burr hole (1.2-mm diameter) was created over the left hemisphere olfactory bulb area with a dental drill (Ram Products, Inc.). A micrometer screw (Worcester Polytechnic Institute) was then inserted into the hole for electrocorticographic recording. For cranial window implantation, another hole was made in the right hemisphere, $4 \mathrm{~mm}$ in diameter centered at a point $3.3 \mathrm{~mm}$ from the midline, and $1.3 \mathrm{~mm}$ from the bregma. For the soft cranial window installation, PDMS (elastomer:curing agent = 10:1, over $24 \mathrm{~h}$ at $75^{\circ} \mathrm{C}$, Sylgard 184, Dow Corning) film-corresponding to a conventional coverslip-was affixed all the way around the edge of the skull with cyanoacrylate glue. PDMS film of 320- to $340-\mu \mathrm{m}$ thickness was used in order to avoid artifacts from pulsation and breathing. During these procedures, extreme caution was taken not to touch the cortical tissue. The applied glue was allowed to dry for more than $10 \mathrm{~min}$. Then, a chamber plate $(8 \mathrm{~mm} \times 10 \mathrm{~mm} \times 1 \mathrm{~mm}$, Narishige Co., Japan $)$ was glued on the same plane with PDMS. The window edge was sealed with dental resin (OA2, Dentkist Inc., Republic of Korea), which was then hardened by UV exposure. The peripheral tissues were disinfected by povidone-iodine (Sung Kwang Pharm, Republic of Korea). After the cranial window implantation surgery, meloxicam $(1 \mathrm{mg} / \mathrm{kg}$, Boehringer Ingelheim, Germany) and Baytril (5 mg/kg, Bayer, Germany) were injected subcutaneously. Animals were supplied with diluted tylenol $(1 \mathrm{ml} / 10 \mathrm{ml}$, Janssen $)$ in water to relieve pain, and they were individually housed in their cages until the experimental sessions. The detailed schematic of the soft cranial window is illustrated in Fig. 1(a).

\subsection{Immunohistochemistry}

Animals at 1 to 6 weeks postsoft cranial window installation were perfused intracardially with saline and fixed with $4 \%$ paraformaldehyde. Brains were then extracted after perfusion and conserved in $4 \% \mathrm{PFA}$ at $4^{\circ} \mathrm{C}$ for postfixation. After $24 \mathrm{~h}$ in PFA, each brain was immersed in $30 \%$ sucrose and $0.1 \%$ sodium azide solution for $24 \mathrm{~h}$, and the media was exchanged. The brains were frozen rapidly in liquid nitrogen and embedded with an optimal cutting temperature compound (Leica Microsystems, Germany). Each brain was coronally sectioned at $40 \mu \mathrm{m}$ by cryostat (Leica CM1950, Leica Microsystems). Brain slices containing the barrel cortex area, 0.62 to $1.94 \mathrm{~mm}$ from the bregma, were selected for detailed analysis. Fluorescent image acquisition was performed by confocal laser scanning microscopy (TCS SP8, Leica Microsystems) with a white light laser (Leica white light laser, Leica Microsystems). The following primary antibodies were used: 4',6-diamidino-2phenylindole (DAPI) (Sigma Aldrich, 1:1000), Iba-1 (a marker of microglia, 1:300, rabbit, Wako, Japan), and GFAP (a marker of reactive astrocyte, 1:300, mouse, Millipore).

\subsection{Cell Counting and Quantification}

The number of GFAP+ cells and Iba+ cells in the region of interest (RoI) was analyzed using IMARIS software (Oxford Instruments, United Kingdom). The cells that overlapped with DAPI signals were counted. In addition, GFAP+ cells that had an almost full shape were counted.

\subsection{Optical Imaging of Cerebral Blood Volume during Whisker Stimulation}

Optical imaging of the intrinsic signal (ORIS) associated with changes in cerebral blood volume (CBV) was performed on animals at 2 and 6 weeks postcranial window surgery. For a single ORIS session, mice from each group were anesthetized by 
(a)
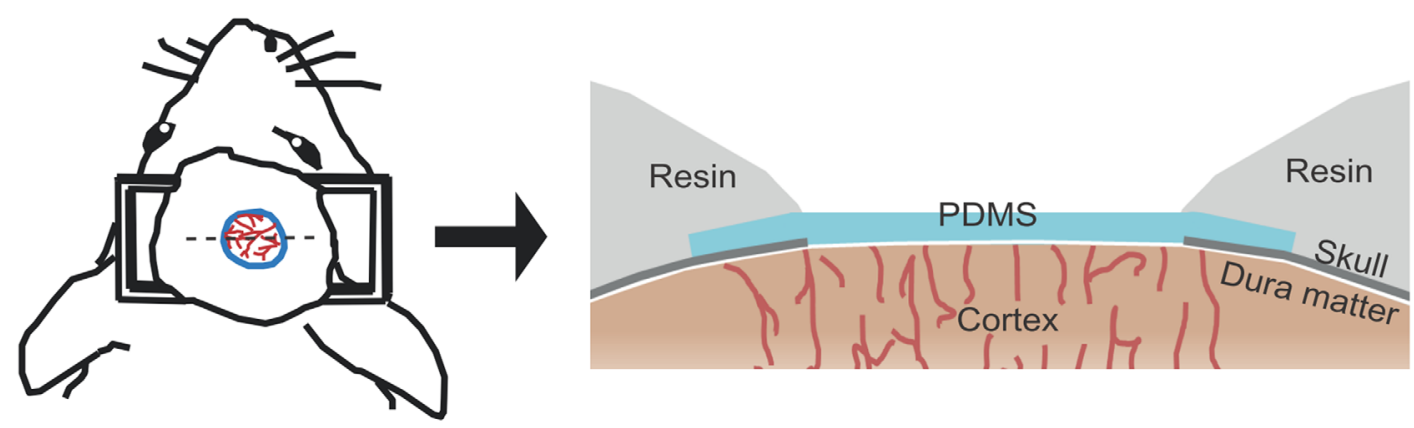

(b)

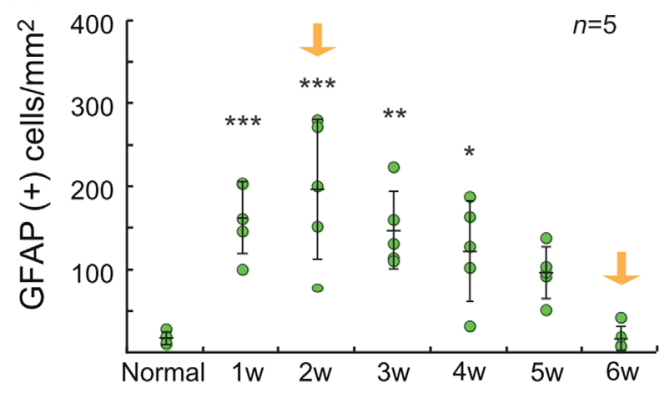

(c)

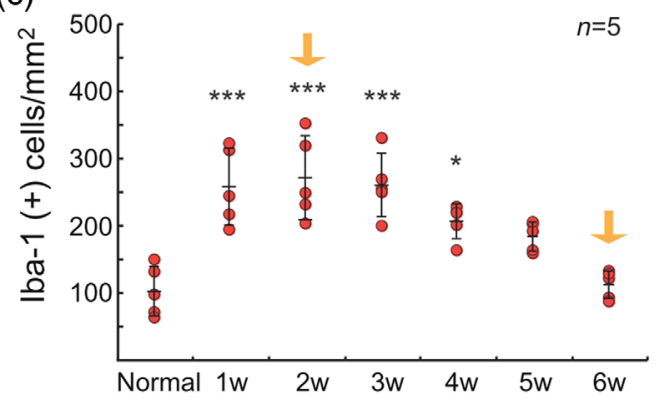

(d)
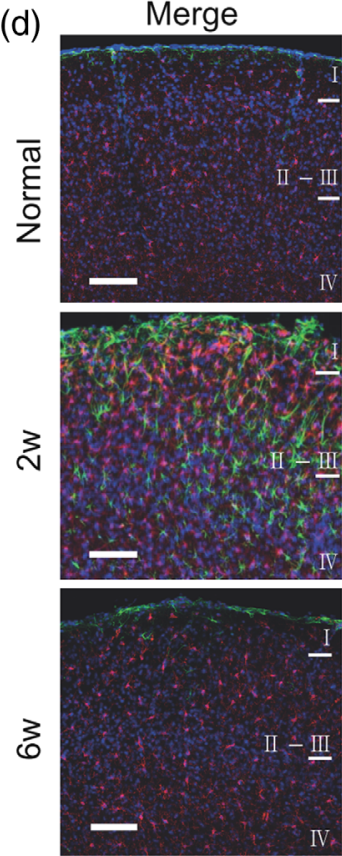

Scale bar: $100 \mu \mathrm{m}$

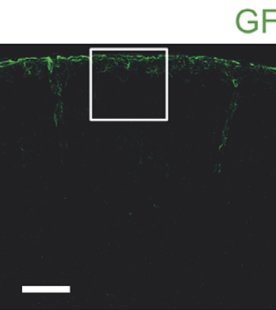

GFAP
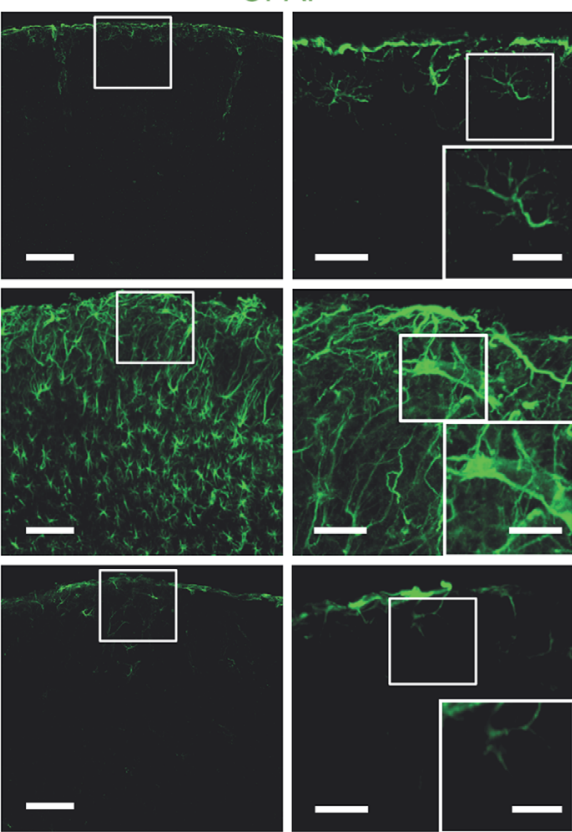

$100 \mu \mathrm{m}$
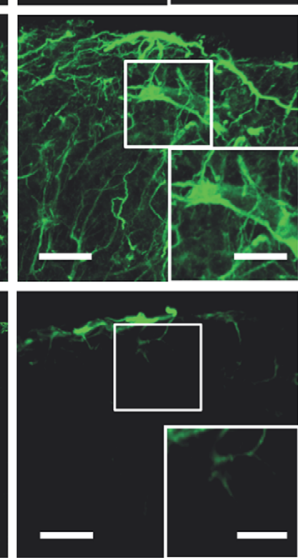

$30 \mu \mathrm{m}$
$15 \mu \mathrm{m} \quad 100 \mu \mathrm{m}$

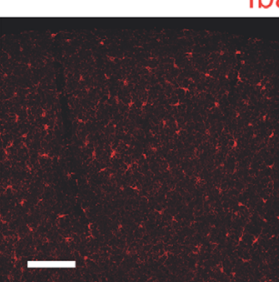

Iba-1
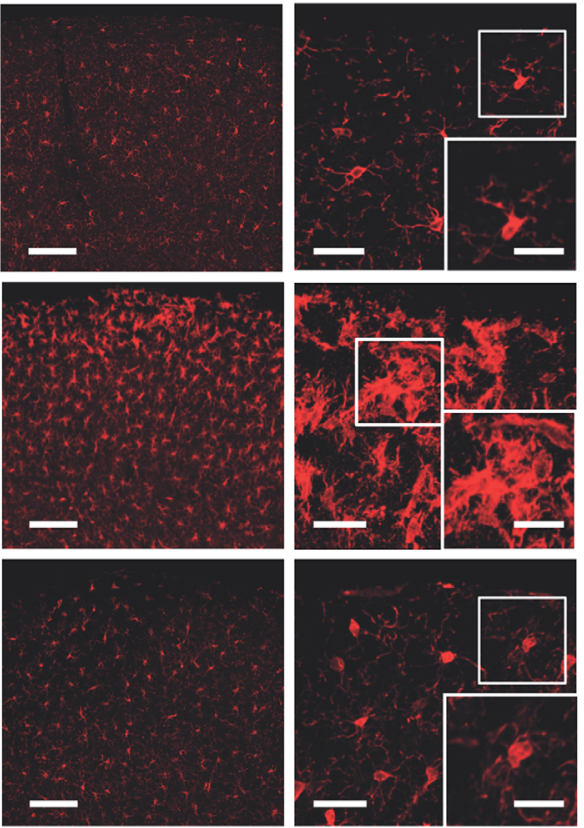

$30 \mu \mathrm{m}$
$15 \mu \mathrm{m}$

Fig. 1 Soft cranial window installation and the time course of reactive astrocyte and microglial expression postsoft cranial window installation. (a) Schematic of the soft cranial window installation with PDMS. (b) and (c) Quantification of GFAP(+) cells (reactive astrocyte) and lba-1(+) cells of the cortex within the soft cranial window area at different postsurgery time points ( $n=5$ in each group). (d) Immunohistochemistry of normal, 2 weeks postsurgery, and 6 weeks postsurgery mice. GFAP levels of astrocytes and microglia were highly activated in the brain of the animals at 2 weeks postsoft cranial window installation $\left({ }^{*} p<0.05\right.$, $\left.{ }^{\star \star} p<0.01,{ }^{\star \star *} p<0.001\right)$.

urethane $(1.3 \mathrm{~g} / \mathrm{kg}$, Sigma-Aldrich) through intraperitoneal injection. Animals were laid on a bed of paper towels and kept warm with a temperature controller (DC temperature controller, FHC). Each animal's head was fixed onto a stereotaxic frame (Narishige Co., Japan). An LED lamp (CLS150LED,
Leica Microsystems) was used for even illumination of the cranial window area. The reflected light signal from the surface was collected by an optical imager system (Imager 3001-Celox, Optical-Imaging, Inc., Jerusalem, Israel) with a CCD camera (PhotonFocus AG, Switzerland). To image CBV, a $570 \mathrm{~nm}$ 
bandpass filter was selected. This wavelength is an isosbestic wavelength sensitive to both oxygenated hemoglobin and deoxygenated hemoglobin; therefore, the measurement reflects total hemoglobin change (i.e., hemodynamic changes). The imaging session consisted of 20-s recordings (with 40 trials). The sampling rate was $10 \mathrm{~Hz}$, and the pixel resolution was $17.7 \times 17.7 \mu \mathrm{m}$. For repeated ORIS imaging, animals were anesthetized with ketamine (Yuhanyanghang, Republic of Korea, $100 \mathrm{mg} / \mathrm{kg}$ ) and xylazine (Rompun, Bayer, Germany, $10 \mathrm{mg} / \mathrm{kg}$ ) through intraperitoneal injection.

For whisker stimulation, the $\mathrm{C} 1$ and $\mathrm{C} 2$ whiskers were stimulated with a piezo actuator (plate benders, Noliac, Denmark) and controlled by a pulse stimulator (Master 9, A.M.P.I., Israel). The stimulation was applied for $4 \mathrm{~s}$. Whole whiskers were stimulated by air puffing from a picospritzer (Picospritzer III, Parker Hannifin) for $4 \mathrm{~s}$ and controlled by a pulse stimulator (Master 9, A.M.P.I., Israel).

\subsection{Data Analysis}

Acquired optical images were analyzed with a MATLAB computer program (Mathworks, Inc.) written to calculate and display the hemodynamic changes. Baseline was defined as the average value obtained from the frames in the 5-s period prior to whisker stimulation. The values from frames obtained after the whisker stimulation were divided by the baseline $(\Delta R / R)$. Spatial mapping of $\Delta R / R$ changes was displayed in a two-dimensional format, and hemodynamic changes were carefully examined. The time course of pixel changes from the selected RoIs $(6 \times 6$ pixels, 1 pixel: $17.7 \mu \mathrm{m} \times 17.7 \mu \mathrm{m})$ was plotted. The maximum $\Delta R / R$ change and the time to the maximum $\Delta R / R$ changes were quantified and averaged within each group. Spatial extent was also obtained by a MATLAB computer program. To quantify spatial extent, we selected the divided frame that the maximum $\Delta R / R$ value of pixel existed in and counted the pixels that had values over $50 \%$ of the maximum value. The shape of the mouse barrel column was taken from Krook-Magnuson et al. ${ }^{14}$

\subsection{In Vivo Electrophysiology Local Field Potential Recording and Data Analysis}

Following ORIS imaging, electrophysiological recordings were performed using tungsten electrodes (FHC) attached to a headstage (Plexon Instruments, Inc.). Three tungsten electrodes with 300 to $500 \mathrm{k} \Omega$ were affixed in a straight line. The central electrode was located on the peak hemodynamic activation site in the $\mathrm{C} 2$ column of the barrel cortex. The other electrodes were located at $625 \mu \mathrm{m}$ rostral and caudal to the center location. Electrodes were stereotaxically inserted into cortex layer 2-3 (300 to $400 \mu \mathrm{m}$ from the surface). Animals were given a $0.1 \mathrm{~ms}$ C2 single whisker deflection by a piezo actuator (plate benders, Noliac) controlled by a pulse stimulator (Master 9, A.M.P.I., Israel). LFPs were acquired using Plexon electrodes (Plexon Instruments, Inc.) and analyzed by software written in MATLAB (Mathworks, Inc.).

\subsection{Inflammatory Cytokine Assay}

The cortex tissue under the cranial window area was homogenized with radioimmunoprecipitation assay buffer containing a $0.01 \%$ phosphatase inhibitor. After centrifuging the sample at $4^{\circ} \mathrm{C}$ and $12700 \mathrm{rpm}$, we performed protein extraction. The
BCA protein assay kit (Pierce Rapid Gold BCA Protein Assay, Thermo Fisher Scientific) was used for measuring protein concentration. Equal amounts of protein $(1500 \mathrm{mg})$ were used to estimate the concentration of inflammatory cytokines [interleukin-1 $\beta$ (IL-1 $\beta$ ), IL-6, and IL-10] by separate enzymelinked immunosorbent assay (ELISA) kits (Mouse IL-1 beta/ IL-1F2 Quantikine ELISA Kit, Mouse IL-6 Quantikine ELISA Kit, Mouse IL-10 Quantikine ELISA Kit, R\&D Systems, respectively).

\subsection{MinoNAC Administration after Soft Cranial Window Installation}

MinoNAC [minocycline (minocycline hydrochloride, SigmaAldrich) and $N$-acetyl-l-cysteine (NAC, Sigma-Aldrich)] was mixed together and prepared as $5 \mathrm{mg} / \mathrm{ml}$ stock solution in phosphate-buffered saline and aliquoted. It was preserved frozen at $-80^{\circ} \mathrm{C}$. After soft cranial window installation, mice were administered minoNAC intraperitoneally (minocycline $22.5 \mathrm{mg} / \mathrm{kg}$, NAC $75 \mathrm{mg} / \mathrm{kg}$ ) every day for 3 days postsoft cranial window surgery. ORIS imaging and LFP recording were performed at 2 weeks postsurgery under urethane anesthesia. C2 whiskers were stimulated for $4 \mathrm{~s}$ by a piezo actuator (plate benders, Noliac) and controlled by a pulse stimulator (Master 9, A.M.P.I., Israel). Experimental procedures for ORIS and LFP recording were the same as those described above.

\subsection{Statistical Analysis}

The Wilcoxon signed rank test was used to compare $\Delta R / R$ changes at 2 and 6 weeks within the same animals. The Mann-Whitney U test was performed for comparison between 2 and 6 weeks groups. Bonferroni correction by ranks was conducted for group comparison of cell quantification data and inflammatory cytokine levels. Independent $T$-test was conducted for comparison of LFP amplitudes between groups.

In the data figures, data are presented as the mean \pm standard error of the mean. However, LFP amplitudes and cell quantification are presented as the mean \pm standard deviation. Statistical significance in the figures is indicated at one of three levels. $* p<0.05, * * p<0.01, * * * p<0.001$.

\section{Results}

\subsection{Immunohistochemical Analysis of Cortical Tissue at Different Time Points Following Soft Cranial Window Installation}

Each week, for up to 6 weeks after the soft cranial-window installation surgery, brains from a subset of mice that had undergone the procedure were perfused and prepared for immunohistochemical analysis. The samples were stained for GFAP, a reactive astrocyte marker, ${ }^{15}$ and Iba-1, a microglia marker related to the brain's innate immune response, ${ }^{16,17}$ as shown in Fig. 2(a).

The immunohistochemical results indicated that at 2 weeks after surgery, both GFAP and lba- 1 showed the highest activity level [i.e., rapid increases in the number of activated astrocytes and microglia; Fig. 1(b)]. After this point, the number of activated cells gradually decreased to the level of normal control mice by 6 weeks after installation of the soft cranial window. All $p$ values are shown in Tables 1 and 2 .

In particular, the microglia in tissues obtained at 1 and 2 weeks postsurgery were highly clustered at the cortex surface, 


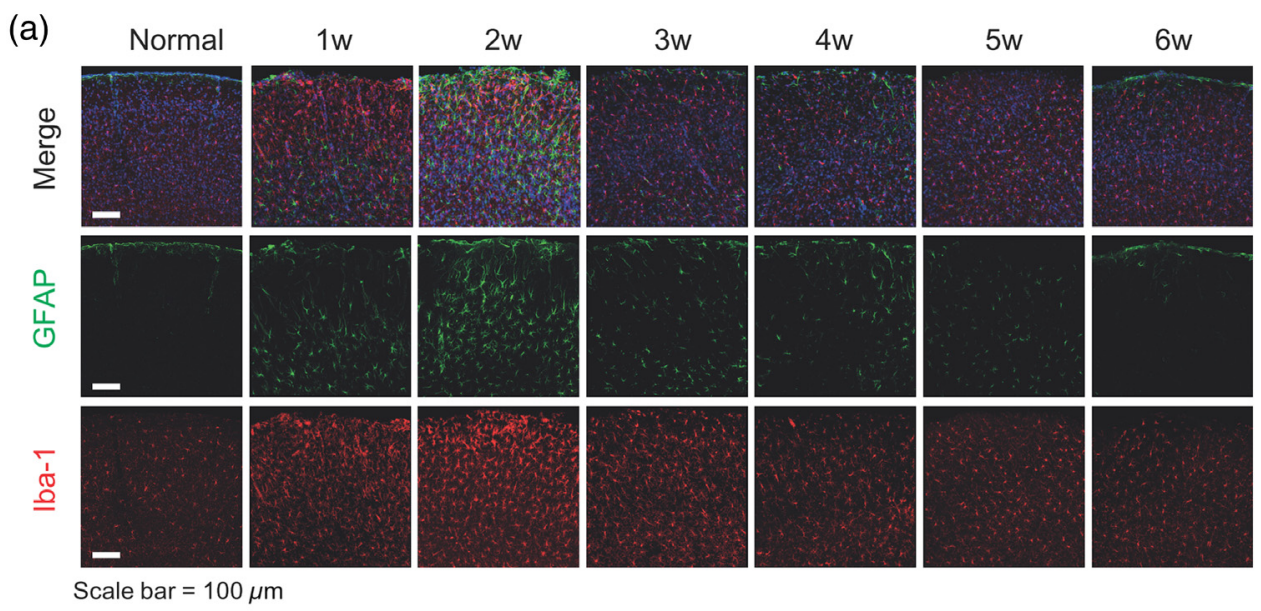

(b)

DAPI GFAP Iba-1
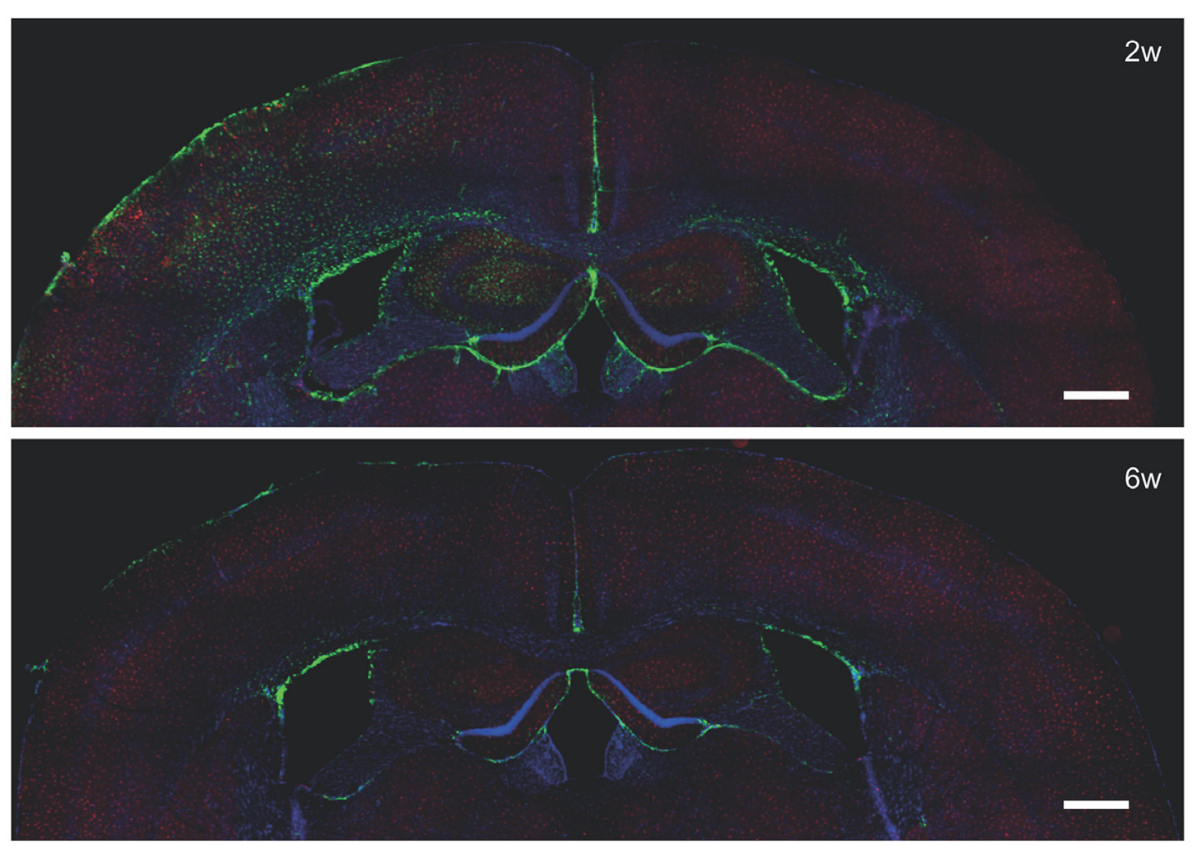

Scale bar $=500 \mu \mathrm{m}$

Fig. 2 The expressions of reactive astrocytes and microglia. (a) Immunohistochemistry of reactive astrocytes and microglia at 1 -week interval from 1 to 6 weeks in normal mice and soft window installation mice. (b) Immunohistochemistry of whole hemispheres at 2 and 6 weeks postsoft window installation mice.

a similar pattern to that of microgliosis. ${ }^{18}$ This clustering was not observed at other postoperative time points or in normal mice. Differential morphological features of microglia were also noticed relative to the postoperative period. Microglia in mice 2 weeks postsurgery were in a highly activated form, known as the amoeboid form, with increased size of soma and disappearance of branches. At 6 weeks postinstallation, the microglia had returned to the same morphology as that found in control animals [Fig. 1(d)]. Based on this immunohistochemical analysis, we focused on the outcomes at two time points - 2 and 6 weeks postoperatively-to examine the effect of soft cranial window installation on hemodynamic and neuronal activation over time.

Reactive astrocyte expression showed a pattern quite similar to the microglia. Morphology of reactive astrocyte has been shown to differ with physiological condition. ${ }^{19}$ In mice at
2 weeks postsurgery, the morphology of reactive astrocytes was much enlarged like the phagocytic form and showed features like astrogliosis. However, at 6 weeks after window installation, the expression level of reactive astrocytes was similar to that of normal animals [Fig. 1(d)]. The expression of reactive astrocyte and microglia in whole hemispheres at 2 and 6 weeks is shown Fig. 2(b).

\subsection{Altered Hemodynamic Changes during Whisker Stimulation at 2 and 6 Weeks Postsoft Cranial Window Surgery}

Hemodynamic changes during whisker stimulation were compared between 2 and 6 weeks postsoft cranial window installation within the same animals when the whole whisker pad was stimulated with air-puffing [Fig. 3(a)]. $\Delta R / R$ changes at 2 weeks 
Park et al.: Longitudinal study of hemodynamics and dendritic membrane...

Table 1 The $p$ value of GFAP (+) expression between groups of normal, at 1 to 6 weeks postsoft window installation.

\begin{tabular}{|c|c|c|c|c|c|c|c|}
\hline GFAP & Normal & 1 week & 2 weeks & 3 weeks & 4 weeks & 5 weeks & 6 weeks \\
\hline \multirow[t]{2}{*}{ Normal } & & $<0.001$ & $<0.001$ & 0.002 & 0.014 & 0.284 & 1 \\
\hline & & $\star \star \star *$ & $\star \star \star *$ & ** & * & NS & NS \\
\hline \multirow[t]{2}{*}{1 week } & $<0.001$ & & 1 & 1 & 1 & 0.384 & $<0.001$ \\
\hline & $* * *$ & & NS & NS & NS & NS & $* * *$ \\
\hline \multirow[t]{2}{*}{2 weeks } & $<0.001$ & 1 & & 1 & 1 & 0.162 & $<0.001$ \\
\hline & $\star \star \star *$ & NS & & NS & NS & NS & $* * *$ \\
\hline \multirow[t]{2}{*}{3 weeks } & 0.002 & 1 & 1 & & 1 & 1 & 0.001 \\
\hline & ** & NS & NS & & NS & NS & ** \\
\hline \multirow[t]{2}{*}{4 weeks } & 0.014 & 1 & 1 & 1 & & 1 & 0.005 \\
\hline & * & NS & NS & NS & & NS & ** \\
\hline \multirow[t]{2}{*}{5 weeks } & 0.284 & 0.384 & 0.162 & 1 & 1 & & 0.117 \\
\hline & NS & NS & NS & NS & NS & & NS \\
\hline \multirow[t]{2}{*}{6 weeks } & 1 & $<0.001$ & $<0.001$ & 0.001 & 0.005 & 0.117 & \\
\hline & NS & $\star \star \star *$ & $\star \star \star \star$ & $\star *$ & $\star *$ & NS & \\
\hline
\end{tabular}

Note: NS, not significant.

${ }^{*} p<0.05$.

${ }^{* *} p<0.01$.

${ }^{* * *} p<0.001$.

Table 2 The $p$ value of Iba-1(+) expression between groups of normal, at 1 to 6 weeks postsoft window installation.

\begin{tabular}{|c|c|c|c|c|c|c|c|}
\hline Iba-1 & Normal & 1 week & 2 weeks & 3 weeks & 4 weeks & 5 weeks & 6 weeks \\
\hline \multirow[t]{2}{*}{ Normal } & & $<0.001$ & $<0.001$ & $<0.001$ & 0.011 & 0.096 & 1 \\
\hline & & $* \star *$ & $* \star *$ & 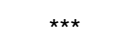 & * & NS & NS \\
\hline \multirow[t]{2}{*}{1 week } & $<0.001$ & & 1 & 1 & 1 & 0.202 & $<0.001$ \\
\hline & $* * *$ & & NS & NS & NS & NS & $* * *$ \\
\hline \multirow[t]{2}{*}{2 weeks } & $<0.001$ & 1 & & 1 & 0.462 & 0.61 & $<0.001$ \\
\hline & $* * *$ & NS & & NS & NS & NS & $* * *$ \\
\hline \multirow[t]{2}{*}{3 weeks } & $<0.001$ & 1 & 1 & & 1 & 1 & $<0.001$ \\
\hline & $\star \star \star *$ & NS & NS & & NS & NS & $\star \star \star *$ \\
\hline \multirow[t]{2}{*}{4 weeks } & 0.011 & 1 & 0.462 & 1 & & 1 & 0.03 \\
\hline & * & NS & NS & NS & & NS & * \\
\hline \multirow[t]{2}{*}{5 weeks } & 0.096 & 0.202 & 0.61 & 1 & 1 & & 0.242 \\
\hline & NS & NS & NS & NS & NS & & NS \\
\hline \multirow[t]{2}{*}{6 weeks } & 1 & $<0.001$ & $<0.001$ & $<0.001$ & 0.03 & 0.242 & \\
\hline & NS & $\star * *$ & $* * *$ & $\star * \star$ & * & NS & \\
\hline
\end{tabular}

Note: NS, not significant.

${ }^{*} p<0.05$.

${ }^{* * *} p<0.001$. 
(a)

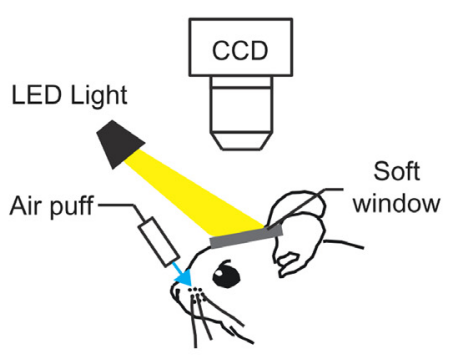

(c)

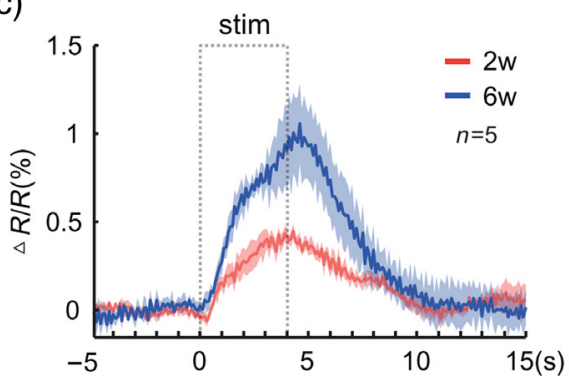

(b)

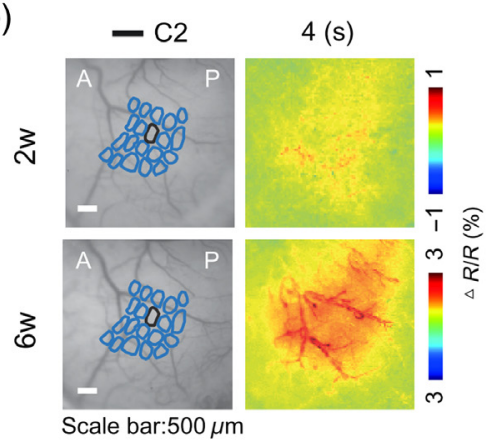

(d)

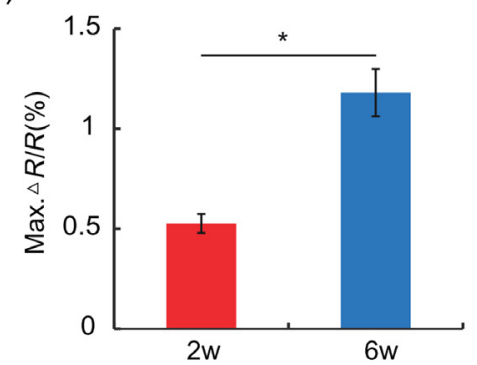

Fig. 3 Hemodynamic signal change $(\Delta R / R)$ within animals at 2 and 6 weeks postsoft cranial window installation. (a) Optical recording of intrinsic signal (ORIS) set up with whole whisker stimulation (4 $\mathrm{s}$ ) by air puff. A 570-nm bandpass filter was used. (b) Spatial activation map of ORIS imaging for whole-whisker stimulation in an animal at 2 or 6 weeks postsoft cranial window installation. (c) The graph of $\Delta R / R$ change of within animals at 2 and 6 weeks postsoft cranial window installation $(n=5)$. (d) The peak value of $\Delta R / R$ change changes within animals at the 2 and 6 weeks postsoft cranial window installation $(n=5 ; p$ value $=0.0431)$.

postsurgery were smaller than those at 6 weeks [maximal $\Delta R / R ; 2$ weeks: $0.53 \pm 0.05 \%, 6$ weeks: $1.18 \pm 0.12 \%$, $p$ value $=0.04, n=5$ in both cases; Figs. 3(b)-3(d)]. There is no significant difference between vessel diameters (at resting state) between at 2 and at 6 weeks postsurgery (see Sec. 5.1).

In addition, when a single whisker $(\mathrm{C} 2)$ was stimulated for $4 \mathrm{~s}$ with a piezo actuator [Fig. 4(a)], $\Delta R / R$ changes at 2 weeks postsurgery were smaller than those at 6 weeks [maximal $\Delta R / R: \quad 2$ weeks $=0.53 \pm 0.14 \%, \quad 6$ weeks $=1.19 \pm 0.14 \%$, $p$ value $<0.001, n=10$ in both cases; Figs. 4(b) and 4(c)]. After acute surgery of soft cranial window installation, hemodynamic change during whisker stimulation was lower than each of those between 2 and 6 weeks groups (see Sec. 5.5; peak value of $\Delta R / R$ change: acute $=0.31 \pm 0.02 \%, 2$ weeks $=$ $0.53 \pm 0.14 \%, 6$ weeks $=1.19 \pm 0.14 \%, p$ value $<0.001)$. These experiments showed that surgical process can affect hemodynamic response immediately and that at 2 weeks postsurgery, the hemodynamic responses were still significantly reduced compared with those at 6 weeks.

In order to verify the spatial extent of hemodynamic responses upon single-whisker stimulation, the spatial extent map of $\Delta R / R$ change was generated. As described in Sec. 2, the pixel values above the $50 \%$ of the peak responses were counted and reflected in a spatial extent map. The spatial extents at 2 weeks were broader than those obtained at 6 weeks $(2$ weeks $=1163.4 \pm 309.49,6$ weeks $=393.4 \pm 33.42$, $p$ value $=0.04)$, suggesting that soft cranial window installation may affect the focality of hemodynamic responses of the barrel cortex [Figs. 4(e) and 4(f)]. In addition, we calculated the mean responses of the $1.06 \mathrm{~mm} \times 2.5 \mathrm{~mm}$ area, which have a peak hemodynamic response. The hemodynamic responses were significantly different between the two groups, as expected, and $\max \Delta R / R$ change of the 6 weeks group was significantly higher than that of the 2 weeks group (see Sec. 5.2; 2 weeks: $0.34 \pm 0.03$, 6 weeks: $0.56 \pm 0.01, p$ value $>0.001 ; n=10$ in each group). In case of change of cerebral blood flow (CBF), the 2 weeks group tended to have smaller $\triangle \mathrm{CBF}$ than the 6 weeks groups. However, there is no significant difference for maximal $\Delta$ CBF between groups (see Sec. 5.3; 2 weeks: $5.53 \pm 1.24 \%$, 6 weeks: $8.95 \pm 3.57 \%, p$ value $=0.132 ; n=6$ in each group).

To confirm this, single $\mathrm{C} 1$ and $\mathrm{C} 2$ whiskers were activated sequentially for $4 \mathrm{~s}$, and the spatial extents of $\Delta R / R$ were generated. In 2-week postoperative mice, the spatial extents of the $\mathrm{C} 1$ and $\mathrm{C} 2$ columns were not adequately separated. By contrast, in animals at 6 weeks postsurgery, the separation of each whisker column was clear, suggesting better focalization of evoked-hemodynamic signals [Fig. 4(g)].

\subsection{Differential Dendritic Potential Changes during Whisker Stimulation after Soft Cranial Window Installation}

In order to investigate whether the decreased evoked-hemodynamic signal in mice at 2 weeks postsurgery was associated with decreased neuronal activity, dendritic membrane potentials from a single whisker column were measured electrophysiologically. Three LFP recording electrodes were inserted directly through the soft cranial window into the center of the $\mathrm{C} 2$ column and into areas rostral and caudal to the first. The electrodes were $600 \mu \mathrm{m}$ apart from each other. The $\mathrm{C} 2$ column was identified using in vivo optical recording of intrinsic signals [Fig. 5(a)].

At 2 weeks postsurgery, whisker-evoked LFP traces at the center of $\mathrm{C} 2$ were not significantly different from the ones 
(a)
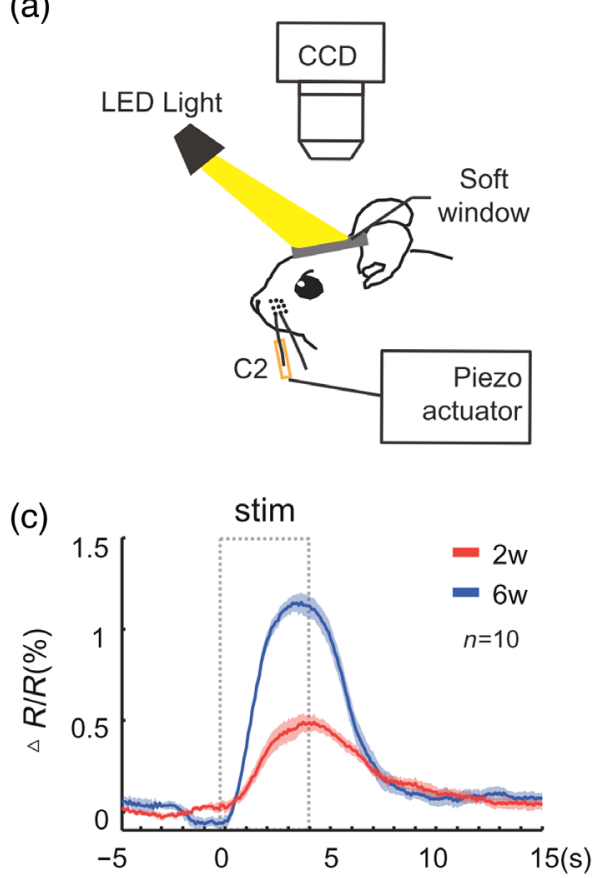

(e)

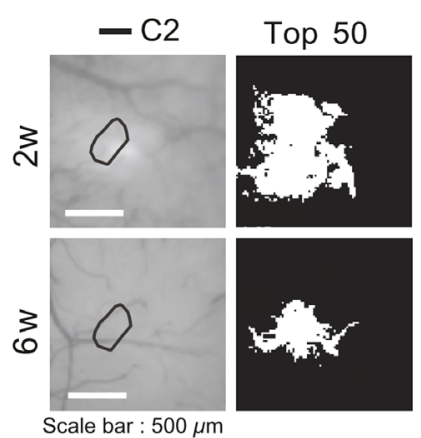

(g)

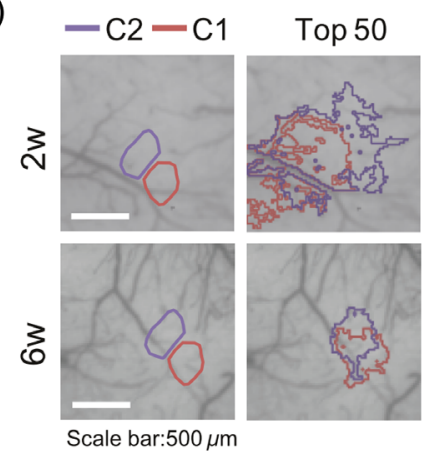

(b)
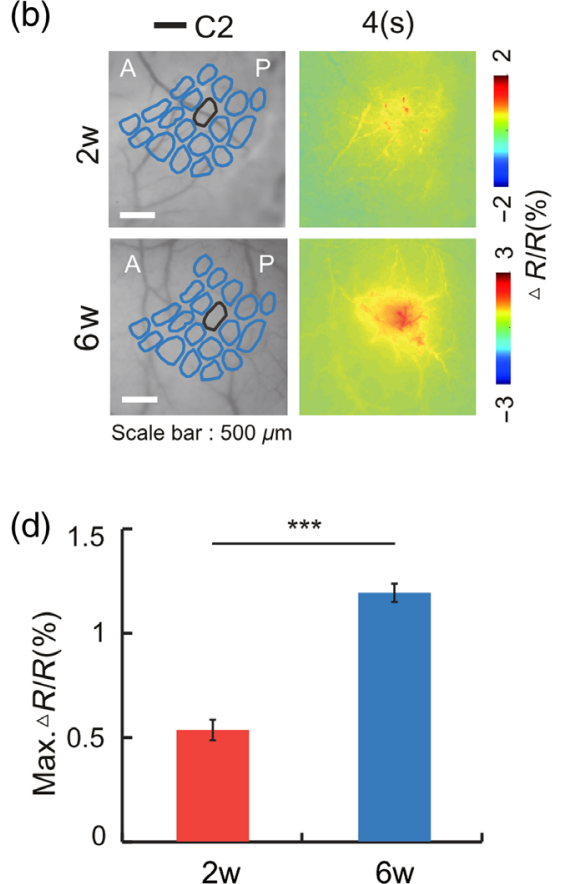

(f)
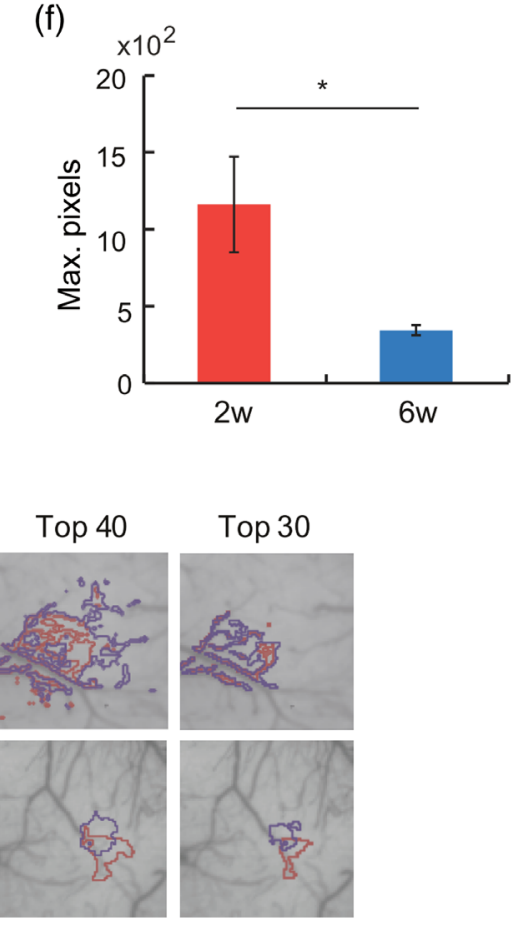

Fig. 4 Hemodynamic change upon piezo stimulus of mice at 2 or 6 weeks postsoft cranial window implantation. (a) Optical recording of intrinsic signal (ORIS) set up with single-whisker stimulation (C2 whisker, 4 s) by piezo actuator. A 570-nm bandpass filter was used. (b) Spatial activation map of ORIS imaging for single-whisker stimulation. (c) Graph of $\Delta R / R$ change $(n=10$ in each group). (d) Peak value of $\Delta R / R$ change ( $n=10$ in each group, $p<0.001)$. (e) Spatial extent map of $\Delta R / R$ change at the activated site following piezo stimulus. The spatial extent map described the areas that have $\Delta R / R$ change values within $50 \%$ of the peak response value of $\Delta R / R$ in whole frames. (f) Pixel counts in the spatial extent maps (pvalue $=0.03546)$. (g) The spatial extent map of $\Delta R / R$ change of $C 1$ and $C 2$ whisker piezo stimulation of an animal 2 and 6 weeks postsoft cranial window installation (Top $50=$ within $50 \%$ of the peak response value of $\Delta R / R$, top $40=$ within $40 \%$ of the peak response value of $\Delta R / R$, top $30=$ within $30 \%$ of the peak response value of $\Delta R / R)$. 
(a)

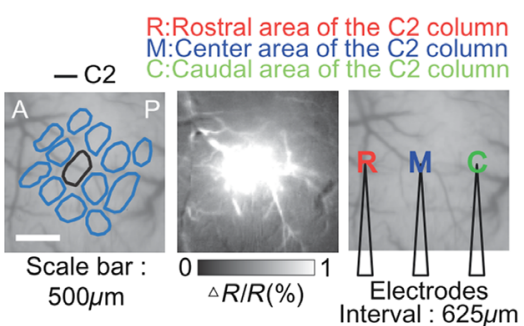

(b)
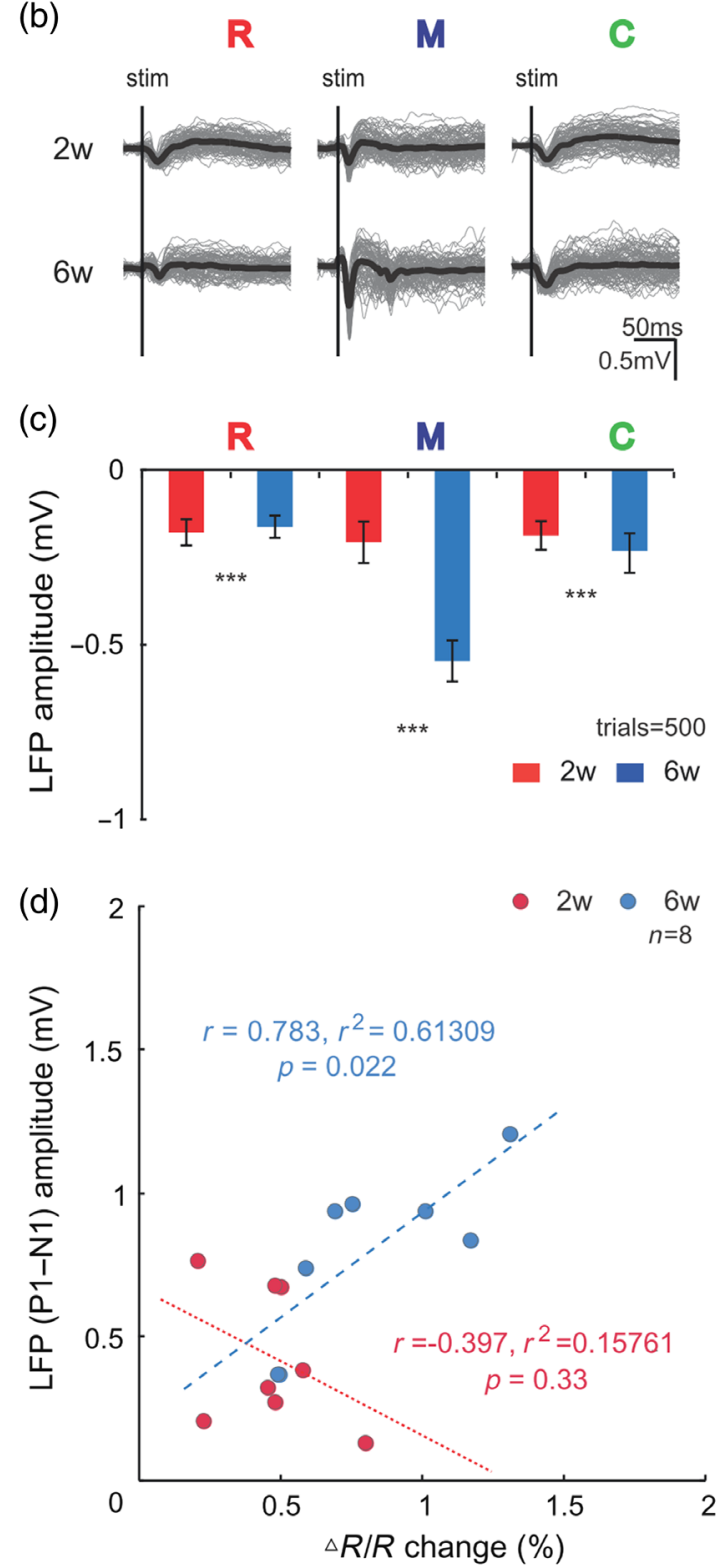

Fig. 5 LFP at 2 and 6 weeks postsoft cranial window installation. (a) In vivo LFP recording set up with $\mathrm{C} 2$ whisker single deflection. Three electrodes recorded LFPs simultaneously in the center of the $\mathrm{C} 2$ column and in both the rostral and caudal directions. (b) LFP following C2 whisker single deflection (trials $=100$ ). (c) Peak amplitude of LFP following C2 whisker single deflection (trials $=500$, all $p$ values $<0.001$ ). (d) Correlation of LFP amplitude in center of column and the peak value of CBV change.

recorded at the neighboring electrodes [Fig. 5(b)]. All three LFPs showed similar levels of cortical activity. On the other hand, at 6 weeks postsurgery, whisker-evoked LFP traces at the center of $\mathrm{C} 2$ were significantly different from the other locations, that is, LFPs from the center of $\mathrm{C} 2$ column showed significantly higher amplitude. When the LFPs obtained at 2 and 6 weeks postsurgery are compared, the amplitude of center LFPs at 6 weeks is significantly higher than those at 2 weeks. LFPs from the rostral and caudal areas showed no significant difference between the two postoperative groups [trials $n=$ 500, R: 2 weeks $=-0.18 \pm 0.04,6$ weeks $=-0.16 \pm 0.03$, M: $\quad 2$ weeks $=-0.21 \pm 0.06, \quad 6$ weeks $=-0.55 \pm 0.06$, C: $\quad 2$ weeks $=-0.19 \pm 0.04, \quad 6$ weeks $=-0.23 \pm 0.06$, all $p$ values $<0.001$; Fig. 5(c)]. The LFP amplitude from acute surgery was similar with that of the 2 weeks and smaller than that of 6 weeks group (acute $=-0.20 \pm 0.04$, 2 weeks $=-0.20 \pm 0.05,6$ weeks $=-0.54 \pm 0.05, p$ value: acute versus 2 weeks $=0.003$, acute versus 6 weeks, 2 weeks versus 6 weeks $=p$ value $>0.001$; trials $=500$ in each group; see Sec. 5.5). Overall, the tuning of cortical activity for the $\mathrm{C} 2$ column was much stronger at 6 weeks after soft cranial surgery.

This less shapely tuned whisker column phenomenon also appeared in the spatial extents map of hemodynamic signals at 2 weeks postsurgery. Therefore, we plotted the evoked-hemodynamic changes against LFPs at the center of $\mathrm{C} 2$ column at both 2 and 6 weeks following PDMS surgery and calculated the correlation between the peak value of $\Delta R / R$ changes and LFPs amplitude by measuring the magnitude between the trough of the first negative peak and the peak of the first positive deflection. The $R$-value for the correlation of the factors was significantly higher in the 6 weeks postsurgery group [2 weeks: $n=8$, 6 weeks: $n=8$; Fig. 5(d)]. Two animal groups underwent similar anesthetic levels (see Sec. 5.4).

\subsection{Increased Interleukin $1 \beta$ Levels at 2 Weeks Postsoft Cranial Window Installation}

To determine the proinflammatory cytokine expression level associated with soft cranial window surgery, we measured IL- $1 \beta$ and IL- 6 of the cortex in the soft cranial window area. We also measured IL-10 as anti-inflammatory cytokine. An ELISA was used for these measurements. The expression of $\mathrm{IL}-1 \beta$ was higher at 2 weeks postsurgery than at either 6 weeks postsurgery or in normal controls (normal: $6.18 \pm 0.89$, 2 weeks: $15.5 \pm 2.53,6$ weeks: $5.89 \pm 1.87, p$ value: normal versus 2 weeks $=0.002, \quad 2$ weeks versus 6 weeks $=$ $p>0.001)$. IL- $1 \beta$ expression at 6 weeks was not significantly different from that of the normal group $[n=5$ for each group; Fig. 6(a)]. IL-6 expression showed no significant difference among the groups [normal: $13.80 \pm 1.23,2$ weeks: $12.61 \pm 0.85$, 6 weeks: $14.70 \pm 1.34$; Fig. 6 (b)]. IL-10 expression in the cortex of the soft cranial window area was similar in the 2 weeks postsurgery group and normal group [normal: $3.26 \pm 0.88,2$ weeks: $4.74 \pm 0.74,6$ weeks: $7.52 \pm 0.52$, $p$ value: normal versus 6 weeks $=0.004 ; n=5$ in each group; Fig. 6(c)].

In the case of expression of IL- $1 \beta$, the animals after acute surgery also expressed high levels of IL- $1 \beta$, such as animals at 2 weeks postsurgery. The expression of IL- $1 \beta$ of the animals injected only with urethane was not significantly different from the normal control and 6 weeks group [Fig. 5(d); normal: $6.76 \pm 0.89$, urethane: $5.16 \pm 0.99$, acute: $14.24 \pm$ 2.25, 2 weeks: $15 \pm 2.53$, MinoNac. $2 w: 6.62 \pm 0.91,6$ weeks: $5.86 \pm 1.87, p$ value in Table $3 ; n=5$ in each group]. 

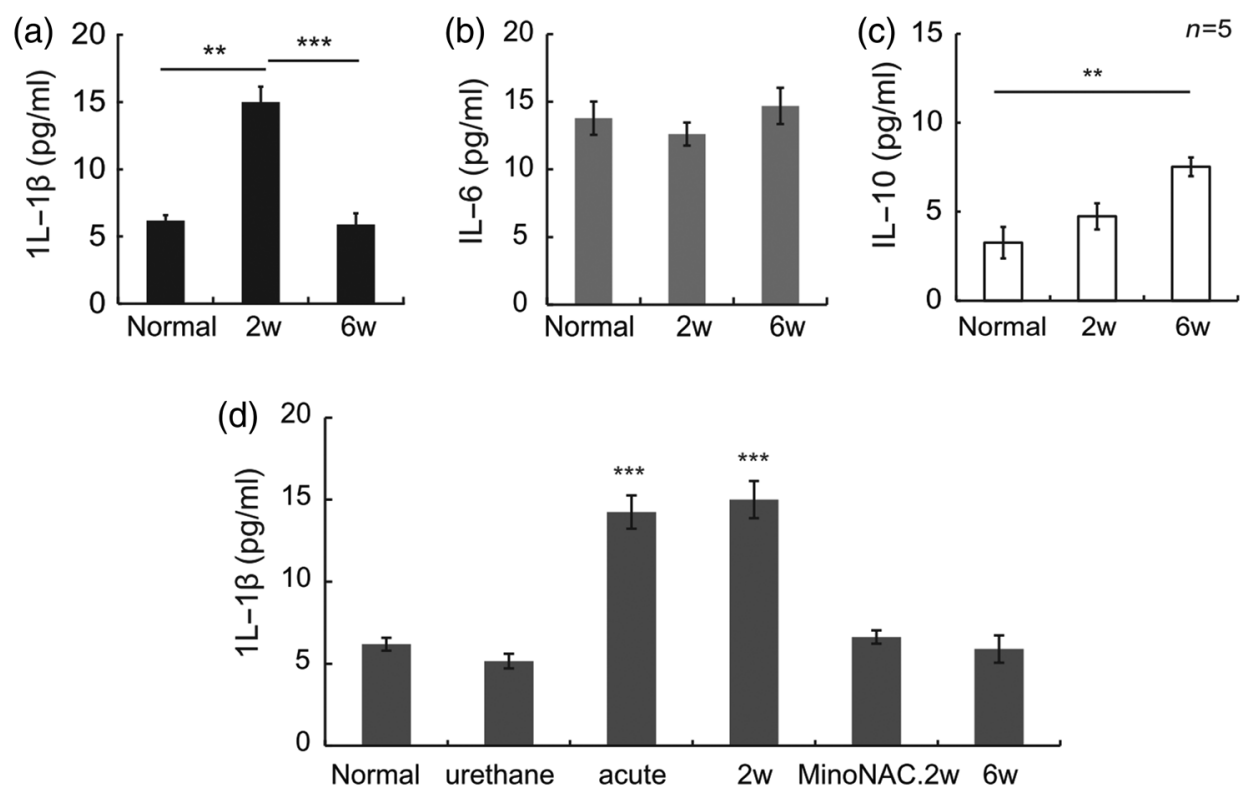

Fig. 6 Expression levels of inflammatory cytokines at 2 or 6 weeks postsoft cranial window installation. (a) Expression of IL-1 $\beta$ ( $p$ value: normal-2 weeks $=0.006,2$ to 6 weeks $=0.001)$. (b) Expression of IL-6. The expression of IL-6 was not significantly different among the groups. Expression levels IL-10 at 2 or 6 weeks postsoft cranial window installation $(p$ value $=0.004)$. (d) Expression level of IL- $1 \beta$ $(n=5 ; p$ value in Table 3$)$.

Table 3 The $p$ value of expression level of IL-1 $\beta$ between groups of normal, urethane, acute, 2 weeks, MinoNAC.2w, 6 weeks postsoft window installation.

\begin{tabular}{|c|c|c|c|c|c|c|}
\hline IL-1 $\beta$ & Normal & Urethane & Acute & 2 weeks & MinoNAC.2w & 6 weeks \\
\hline \multirow[t]{2}{*}{ Normal } & & 1 & 0.006 & 0.002 & 1 & 0.284 \\
\hline & & NS & $\star *$ & $\star \star$ & NS & NS \\
\hline \multirow[t]{2}{*}{ Urethane } & 1 & & $<0.001$ & $<0.001$ & 0.384 & 1 \\
\hline & NS & & *** & *** & NS & NS \\
\hline \multirow[t]{2}{*}{ Acute } & 0.006 & $<0.001$ & & $<0.001$ & 0.035 & 0.001 \\
\hline & $\star \star$ & *** & & 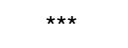 & * & $\star \star *$ \\
\hline \multirow[t]{2}{*}{2 weeks } & 0.002 & $<0.001$ & 1 & & 0.012 & $<0.001$ \\
\hline & $* *$ & $\star \star \star *$ & NS & & * & $* \star *$ \\
\hline \multirow[t]{2}{*}{ MinoNAC.2w } & 1 & 0.389 & 0.035 & 0.012 & & 1 \\
\hline & NS & NS & * & * & & NS \\
\hline \multirow[t]{2}{*}{6 weeks } & 0.284 & 1 & 0.001 & $<0.001$ & 1 & \\
\hline & NS & NS & $\star \star$ & 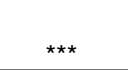 & NS & \\
\hline
\end{tabular}

Note: NS, not significant.

${ }^{*} p<0.05$.

${ }^{* *} p<0.01$.

${ }^{* * *} p<0.001$.

\subsection{Elevated Hemodynamic Response Following minoNAC Administration after Soft Cranial Window Installation Surgery}

Minocycline is a tetracycline antibiotic and known to inhibit the activation and proliferation of microglia. ${ }^{20} \mathrm{~N}$-acetylcysteine is an antioxidant and also used as an antiinflammatory drug. ${ }^{21}$ We mixed these two in order to synergically modulate microglia activation. ${ }^{22,23}$ To test whether the elevation in active microglia of mouse with a PDMS soft cranial window is related with hemodynamic changes, we inhibited neuroinflammation by administering minoNAC to animals immediately following 
(a)

Soft window installation

$\downarrow$

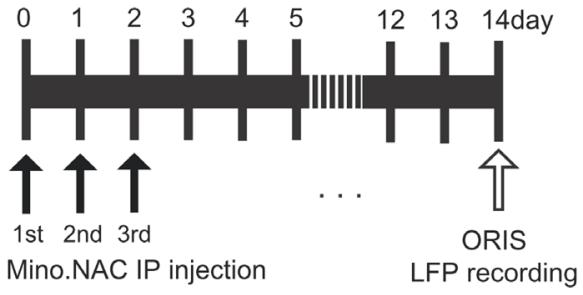

(c)

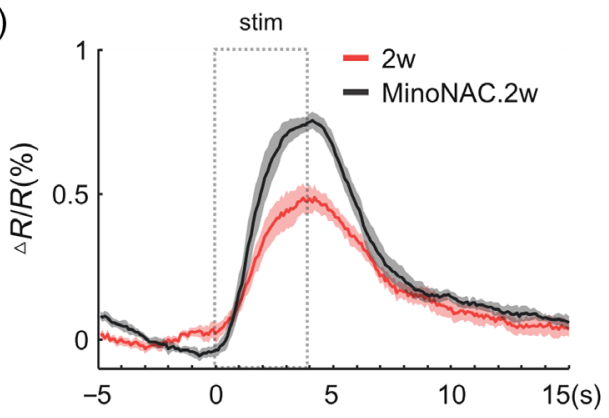

(e)

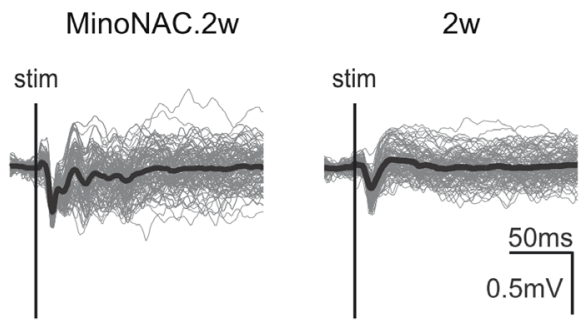

(b)

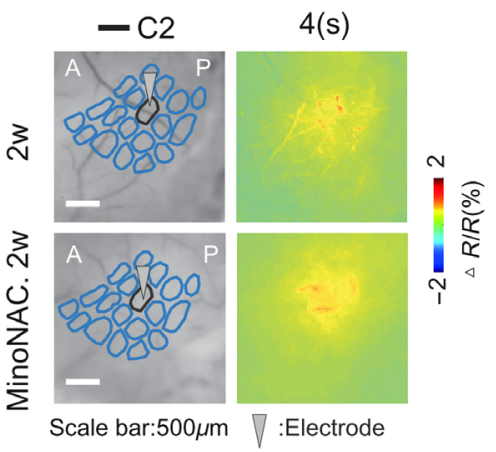

(d)

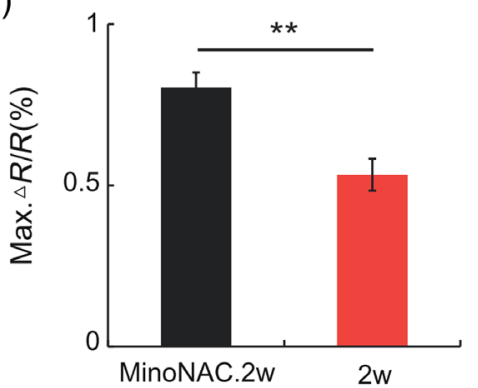

(f)

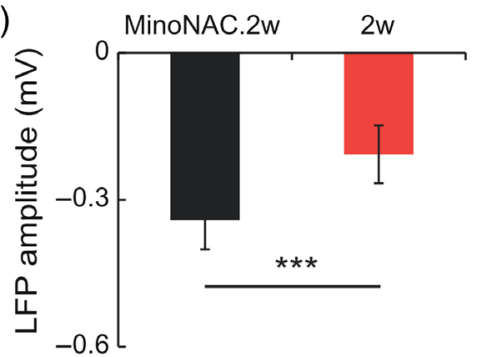

(g)
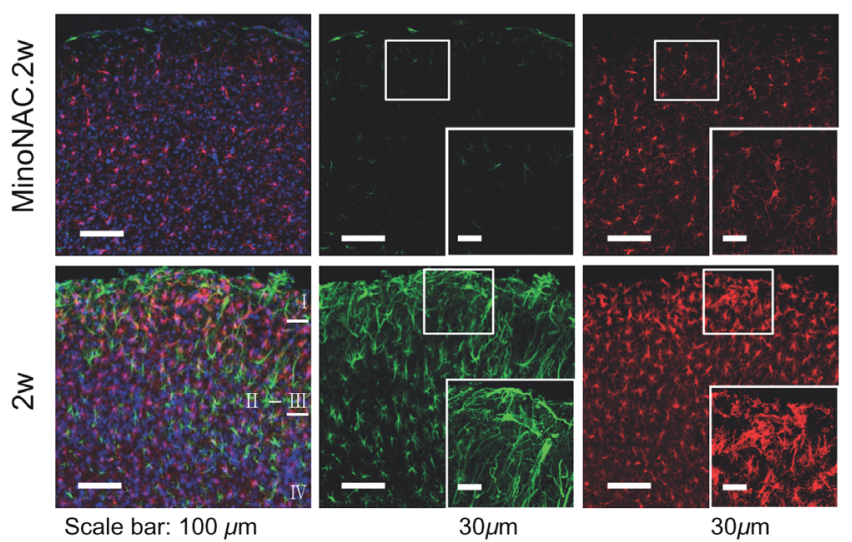

$30 \mu \mathrm{m}$

Fig. 7 Hemodynamic and neural activity from animals with minocycline with NAC administration postsoft cranial window installation. (a) An experimental scheme of the mixture of minocycline with NAC administration for 3 days postsoft cranial window installation. (b) The spatial activation map of ORIS imaging for a single-whisker stimulation $(4 \mathrm{~s})$ in the animal with or without minocycline with NAC at 2 weeks postsoft cranial window installation. (c) A graph of $\Delta R / R$ change at 2 weeks postsoft cranial window installation in mice with or without minocycline plus NAC (minoNAC: $n=6,2$ weeks: $n=10$ ).

(d) The peak value of $\Delta R / R$ changes. (e) LFP following C2 whisker single deflection (trials $=100$ ).

(f) The peak amplitude of LFP following C2 whisker single deflection (trials $=500, p$ value $>0.001$ ).

(g) Immunohistochemistry of reactive astrocyte (green) and microglia (red) of an animal treated with minoNAC. 
soft cranial window installation. Treatment was given every day for 3 days [Fig. 7(a)]. In animals that received minoNAC, whisker evoked-hemodynamic signals were not reduced at 2 weeks postsurgery when compared to the untreated mice 2 weeks [MinoNAC. $2 \mathrm{w}: 0.80 \pm 0.11,2$ weeks: $0.54 \pm 0.07$, $p$ value $=0.005 ;$ MinoNAC: $n=6,2$ weeks: $n=10 ;$ Figs. 7(b) 7(d)]. In addition, amplitude of LFP of received minoNAC animals was higher than the untreated 2 weeks group [MinoNAC.2w: $-0.34 \pm 0.59, \quad 2$ weeks: $0.20 \pm 0.59, \quad p$ value $<0.001$; MinoNAC: $n=6,2$ weeks: $n=10$; Figs. $7(\mathrm{e})$ and 7(f)]. They even maintained low levels of reactive astrocyte and deactivated microglia from IHC compared with the untreated 2 weeks group [Fig. 7(g)] and expressed similar levels of IL$1 \beta$ with normal animals [Fig. 6(d); $n=5, p$ value in Table 3]. These results suggest that neuroinflammation may have a direct effect on decreased hemodynamics and cortical activity at 2 weeks postsurgery.

\section{Discussion and Conclusion}

In this study, we investigated the effects of soft cranial window installation on hemodynamics and neuronal activity over the postoperative recovery period. Soft cranial window installation is an open-skull cranial window that is accompanied by an invasive surgical process and disparate substance unlike skull, such as PDMS. These factors can affect the physiological condition of the brain and, by extension, function of the brain. However, it is still unclear that how the surgical procedure and material used for the window alter the environment and function of the brain. We found that activated microglia and astrocytes reached highest levels at 2 weeks postsoft cranial window installation while these were low levels at 6 weeks postinstallation. IL- $1 \beta$ level was the highest at 2 weeks postinstallation, whereas the level at 6 weeks postinstallation was similar to that of the normal group. Sensory-evoked hemodynamics declined in parallel with the drop in LFP recording from the activated whisker barrel cortex at 2 weeks postsurgery compared to at 6 weeks postinstallation. Interestingly, when minoNAC, an inhibitor of active microglia, was administered immediately after the operation, there was no decline in evoked-hemodynamics and neuronal activity at the 2-week time point. These results suggest a strong link between the activation of glia cells, heightened IL- $1 \beta$, and decreased hemodynamics at 2 weeks after soft cranial window installation.

Our study may provide an important clue for the relationship between microglial activation and hemodynamics and LFP recordings. Microglial immune cells have mobility in the central nervous system (CNS). ${ }^{24}$ Microglia conduct homeostasis surveillance in the resting state and sense pathological change in the CNS and react to that change. If there is an alteration in the brain environment, then microglia shift into an active state-changing its form very quickly, quitting screening, and starting to produce the molecules for brain tissue protection. ${ }^{25}$

In this study, the microglial morphology was obviously different in the brains of the 2 weeks postinstallation mice. At this time point, microglia were missing their branches and had assumed a round shape that looks similar to the amoeboid form. ${ }^{26}$ In addition, during the first 2 weeks after installation, microglia were clustered at the top of the cortical surface. Therefore, it is likely that microglia were activated immediately following the soft cranial window installation. The morphology and clustering behavior of microglia were beginning to recover 3 weeks postsurgery and had returned to the normal state at
6 weeks postsurgery. Activation of microglia could negatively affect neurons by releasing neurotoxic cytokines ${ }^{27}$ and proinflammatory cytokines. ${ }^{28}$ Microglia can affect neuronal function directly by releasing cytokines and prostaglandin, which can modulate the level of neuronal activation in physiological and pathological condition. ${ }^{29}$ Also, inflammatory modulators from activated microglia disrupt the blood-brain barrier $(\mathrm{BBB}) .^{30}$

Therefore, the decreased hemodynamics in the animals 2 weeks following surgery could be simply due to decreased neuronal activation. To examine neuronal activation, we recorded LFPs from the whisker barrel during whisker stimulation. While significantly reduced at the 2 -week time point, the amplitude of LFPs recovered to baseline levels at 6 weeks postsurgery. When we plotted the evoked hemodynamic changes against LFP at both 2 and 6 weeks following PDMS surgery, there was a difference in the slope between the two groups. This change in slope indicates that NVC is affected by the soft cranial window installation procedure itself. Further investigation is needed to find out the mechanism of altered NVC observed 2 weeks after installation of the soft cranial window.

To further investigate the role of microglia, we administrated anti-inflammatory drug, minoNAC. Minocycline is a tetracycline antibiotic that can cross the $\mathrm{BBB}^{31,32}$ and selectively inhibit the M1 polarization of microglia. ${ }^{33}$ Minocycline suppresses the production of proinflammatory cytokines [IL-1 $\beta$, Tumor necrosis factor- $\alpha$ (TNF- $\alpha$ ), and IL-6] by blocking the nuclear translocation of NF- $\kappa \mathrm{B} .{ }^{34} \mathrm{NAC}$ is an antioxidant and used anti-inflammatory drug. Redox-dependent NF- $\kappa \mathrm{B}$ inhibition and suppression of proinflammatory cytokines is anti-inflammatory action of NAC. ${ }^{35}$ Further, minoNAC has a synergized effect to modulate microglia activation. . $2,23^{2}$

In contrast with postsurgery mice that did not receive the antibiotic, animals with repeated doses of minoNAC did not exhibit the 2-week postsurgery reduction in hemodynamic responses and neuronal activity. The anti-inflammatory properties of minoNAC may elevate hemodynamic responses and neuronal activity, even at 2 weeks postsurgery.

Astrocytes are involved in cerebral vascular activity. ${ }^{36}$ One of the cerebral vasculature components surround the blood vessel by an endfoot and form the glial limitans around vessels. ${ }^{37,38}$ Astrocytes can be activated by neuronal activity and give signals to the smooth muscle cells that manage vascular dynamics. ${ }^{39,40}$ In addition, astrocytes can be part of the innate immune response to disease state. ${ }^{41}$ When the brain is damaged, astrocytes become reactive at the injury site ${ }^{42,43}$ and produce proinflammatory cytokines (IL-1 $\beta$, TNF- $\alpha$, and IL-6). ${ }^{17,44}$ Reactive astrocytes also have the ability to affect brain hemodynamics. ${ }^{45}$ In our study, astrocytes also became activated following soft cranial window installation. The time course of astrocytic activation was very similar to the pattern of microglial activation. When astrocytes were reactive, at the 2-weeks postsurgery time point, we observed reduction of hemodynamic signal during sensory stimulation. Then, when the number of reactive astrocytes decreased to the normal levels at 6 weeks postsurgery, animals exhibited higher hemodynamic response than at 2 weeks.

A possible alternative explanation for the study results is an increase in cytokines due to surgery itself. There are previous reports that inflammation and cytokines are increased following open-skull surgery and lead to heightened activation in microglia and astrocytes. ${ }^{4,10}$ Even noninvasive procedures like drilling the skull could generate traumatic brain injury; ${ }^{9}$ therefore, open- 
skull surgery itself would be a critical factor in causing traumatic brain injury-like effects. ${ }^{4,10}$ In another consideration, the effect of open-skull surgery could be heightened by the use of PDMS substances in the soft cranial window. Although PDMS is known to be biocompatible and is used in many implants, materials such as PDMS or PET may boost cytokine production within the biosystem. In a previous study, when PDMS was implanted into the skin of mice, an inflammatory reaction was invoked that peaked at 14 days after the implantation and was sustained for 105 days. Dalu et al. ${ }^{46}$ found that both $\mathrm{IL}-1 \beta$ and TNF- $\alpha$ were at their highest levels at 14 days postsurgery and were sustained for some time. The brain may react in a similar way to PDMS implantation. If so, the altered hemodynamics and neuronal activity from our study could be explained by the effects of cytokines, such as IL- $1 \beta$, produced by PDMS implantation. IL- $1 \beta$ is also released from activated microglia and astrocytes. Indeed, we found elevated IL- $1 \beta$ levels that peaked at 2 weeks postinstallation. Thus, IL- $1 \beta$ could be the main player in altering hemodynamics and neuronal activity in our study. Supporting this inference is a study showing the effects of direct injection of IL- $1 \beta$ on cerebral hemodynamics and neuronal activation. ${ }^{47}$ In that study, animals with a single intrastriatal injection of IL- $1 \beta$ showed decreased hemodynamics within hours of injection. In addition, local tissue oxygenation and LFP recordings also dropped within hours of injection. Their results suggest that IL- $1 \beta$ has an adverse effect on NVC, although the exact mechanism of which the signaling pathways are affected by IL- $1 \beta$ remains unclear.

From our study, we have clear evidence that the soft cranial window installation procedure affects the functional condition of the brain. If the recovery time is too short, the animals undergoing the procedure may show both decreased hemodynamics and neuronal activation. Thus, when research requires the precise mapping of brain function, animals with implanted soft cranial windows should have sufficient recovery time to allow stable data acquisition. With longer rest after cranial window surgery, more heightened CBV and neuronal activity changes are expected during sensory stimulation.

\section{Appendix}

\subsection{Comparison of Blood Vessel Diameters of Resting State between 2 and 6 Weeks Postsoft Window Installation}

In order to verify and compare the diameter of blood vessel in resting state, we measured the baseline diameter of an identical vessel at the 2 and 6 weeks images from our experiment (pixel resolution: 1 pixel: $17.7 \mu \mathrm{m})$. We selected the middle cerebral artery (MCA) as the blood supply source of the barrel cortex for these comparisons. As shown in Fig. 3(a), three vessel segments were selected before and after the bifurcation based on the first bifurcation point of the MCA. The bold line, which has $\sim 100 \mu$ m width, was set perpendicularly to each selected vessel. We quantified five diameters with a parallel shift following the bold line using the "diameter" plugin function of ImageJ. ${ }^{48}$ Thus, we measured 15 diameters from three blood vessels for each individual animal at two time points, i.e., at 2 and 6 weeks postsoft cranial window installation. For the comparison of the measured diameters, we first verified the normality of data through the Shapiro-Wilk test. Based on the normality results, all data were compared by paired $t$-test.

To sum, there is no significant difference between vessel diameters between 2 and 6 weeks postsurgery [Fig. 3(b)]. Thus, the resting state of the vessel diameter was not different between 2 and 6 weeks (Fig. 8) $(2$ weeks $=83.5 \pm 1.84 \%, 6$ weeks $=$ $84.39 \pm 6.65 \%, p$ value: $0.730 ; n=5$ in each group).

\subsection{Comparison of Hemodynamic Responses from the Large Field of View between 2 and 6 Weeks Postsoft-Window Installation}

We calculated the mean responses of more RoIs, $1.06 \mathrm{~mm} \times 2.5 \mathrm{~mm}$ area, which have a peak hemodynamic response (Fig. 9).

\subsection{Comparison of Cerebral Blood Flow (CBF) between 2 and 6 Weeks Postsoft-Window Installation}

For measurement of the local microcirculatory blood perfusion in the capillaries of animal's cortex, a small fiber probe (0.25 mm, 780-nm wavelength laser) connected to a laser Doppler instrument (Periflux system, Perimed, Sweden) was placed at the $\mathrm{C} 2$ barrel column, which was confirmed by ORIS imaging. Piezo stimulation $(10 \mathrm{~Hz})$ delivered to $\mathrm{C} 2$ whiskers was given during $4 \mathrm{~s}$ at $5 \mathrm{~s}$ after onset recording of LDF simultaneously. Laser Doppler instruments were connected with an AC convertor (Plexon Instruments, Inc.) and signals were digitized $(1000 \mathrm{~Hz})$ and recorded. Acquired signals from laser Doppler instrument were analyzed by MATLAB. $1000 \mathrm{~Hz}$ signals were downsampled to $10 \mathrm{~Hz}$ and smoothed by a moving average filter with window span of 10 . The baseline was determined by signals from 1 to $5 \mathrm{~s}$, and all signals were normalized with the baseline. (a)

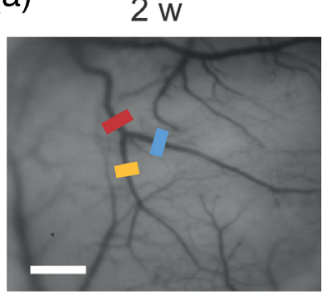

Scale bar : $500 \mu \mathrm{m}$

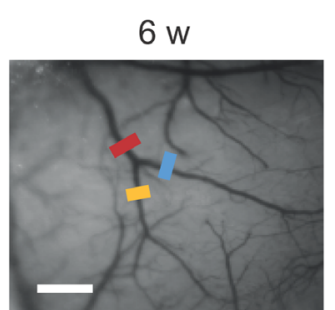

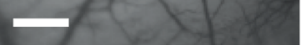

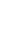

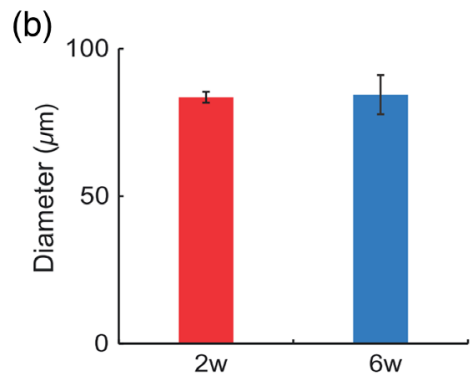

Fig. 8 Blood vessel diameter comparison within a single animal at 2 and 6 weeks postsoft cranial window installation. (a) The images of vessels for the comparison from an animal at 2 weeks (left) and 6 weeks (right) postsoft cranial window implantation. (b) The averaged blood vessel diameters from an animal at 2 and 6 weeks postsoft cranial window implantation. 
(a)
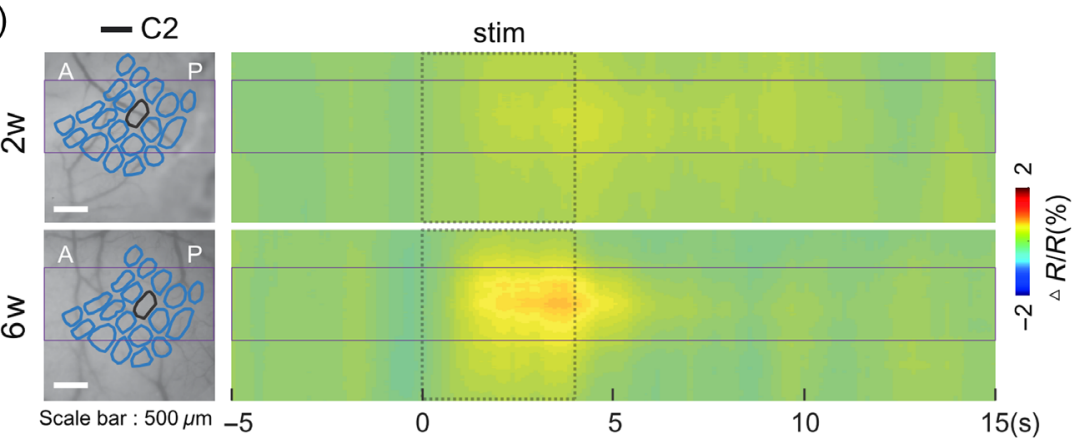

(b)

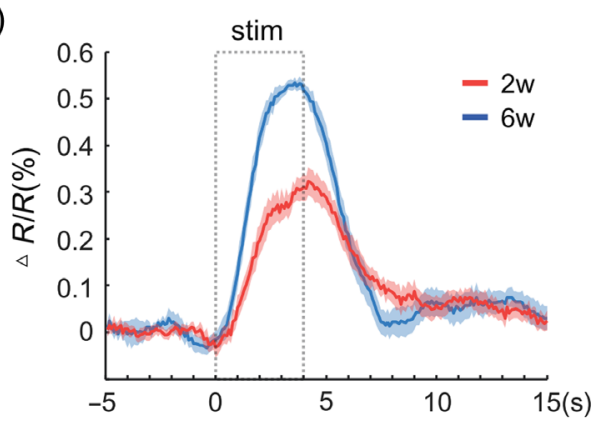

(c)

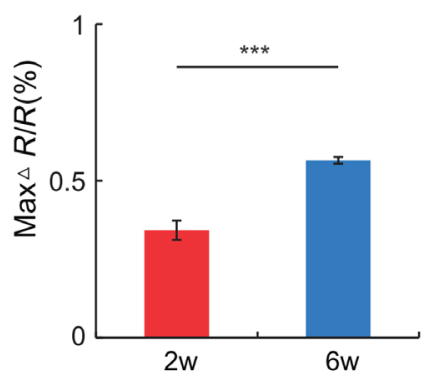

Fig. 9 Hemodynamic responses in $1.06 \mathrm{~mm} \times 2.5 \mathrm{~mm}$ areas of animals at 2 and 6 weeks postsoft cranial window installation. (a) Spatiotemporal map of $\Delta R / R$ change of animal 2 and 6 weeks postsoft window installation. (b) Graph of $\Delta R / R$ change ( $n=10$ in each group). (c) Peak value of $\Delta R / R$ change ( $n=10$ in each group).

(a)

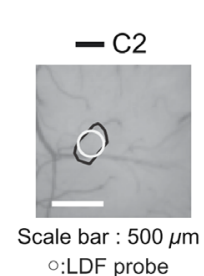

o:LDF probe
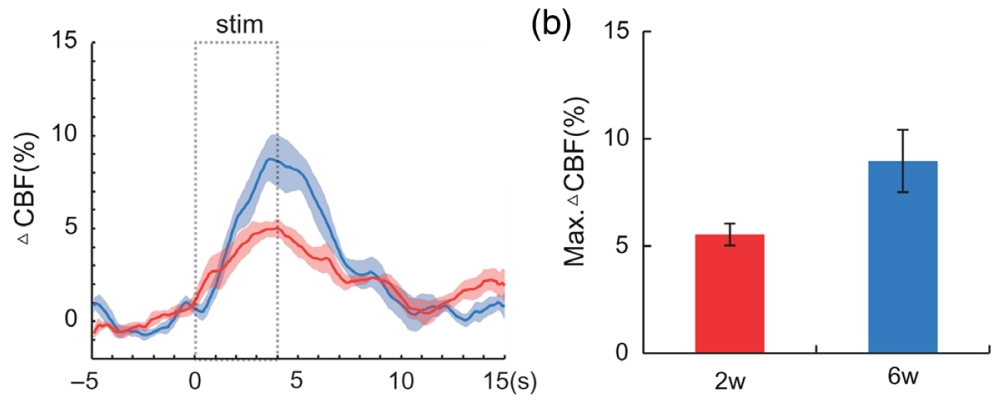

Fig. $10 \mathrm{CBF}$ changes at 2 or 6 weeks postsoft window installation. (a) $\triangle \mathrm{CBF}$ change of animals at 2 and 6 weeks postsoft window installation. (b) peak value of $\Delta$ CBF change of animals at 2 and 6 weeks postsoft window installation ( $n=6$ in each group).

The results were shown that the 2 weeks group tend to have smaller $\triangle \mathrm{CBF}$ than the 6 weeks groups [Fig. 10(a)]. However, there is no significant difference for maximal $\triangle \mathrm{CBF}$ between groups [Fig. 10(b); 2 weeks: $5.53 \pm 1.24,6$ weeks : $8.95 \pm 3.57, p$ value $=0.132 ; n=6$ in each group]. All $\Delta \mathrm{CBF}$ changes of two groups were higher than $1 \%$.

\subsection{Anesthetic Levels of Animals at 2 and 6 Weeks Postsoft Window Installation}

The distribution of power spectral of brain oscillation may reflect the depth of anesthesia. ${ }^{49-51}$ In order to check the level of anesthetic depth during our experiment, we applied fast Fourier transformation (FFT) for spontaneous neuronal activity with no stimulation for $20 \mathrm{~s}$. FFT results showed that there is no difference between two groups in power spectrum [Figs. 11(a) and $11(\mathrm{~b}) ; n=8$ in each group].

\subsection{Acute Assessment of Hemodynamic Responses and Neuronal Activity Immediately Following Soft-Window Installation}

We performed the acute surgery and conducted hemodynamic and neural activity measurements. We followed same experimental protocol as other condition. Although this was a group comparison, hemodynamic change during whisker stimulation was lower than those between 2 and 6 weeks group, respectively [Figs. 12(a), 12(b), and 12(d); peak value of $\Delta R / R$ change : acute $=0.31 \pm 0.02 \%, 2$ weeks $=0.53 \pm$ $0.14 \%, \quad 6$ weeks $=1.19 \pm 0.14 \%, \quad p$ value $<0.001 ; \quad$ acute: $n=7,2$ and 6 weeks: $n=10]$. The LFP amplitude from acute surgery was similar with LFP amplitude from 2 weeks and smaller than that of the 6 weeks group [acute $=$ $-0.20 \pm 0.04,2$ weeks $=-0.20 \pm 0.05,6$ weeks $=-0.54 \pm$ $0.05, p$ value: acute versus 2 weeks $=0.003$, acute versus 

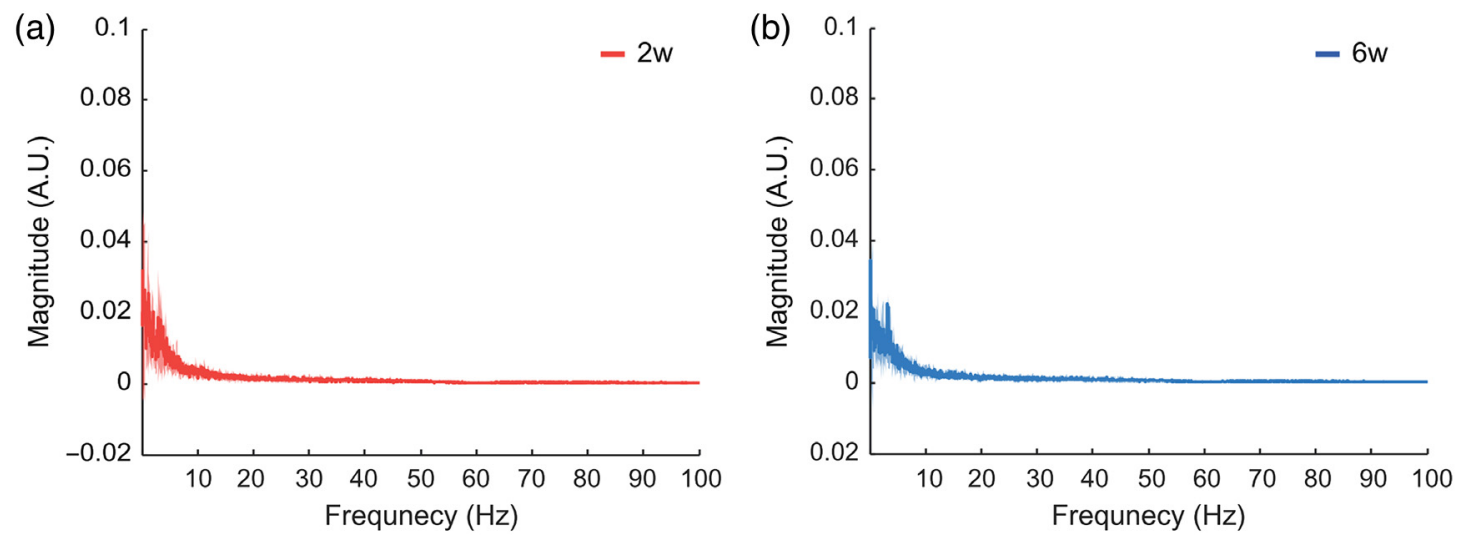

Fig. 11 The power spectrums of spontaneous neuronal activity. (a) Power spectrum of spontaneous neuronal activity of 2 weeks group $(n=8)$. (b) Power spectrum of spontaneous neuronal activity of 6 weeks group $(n=8)$.

(a)

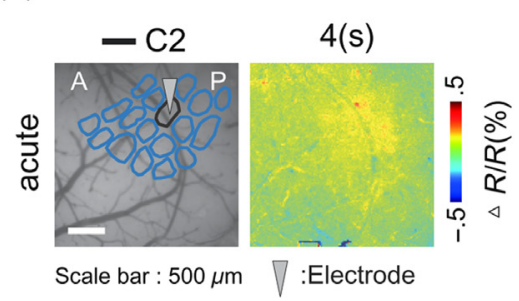

(b)

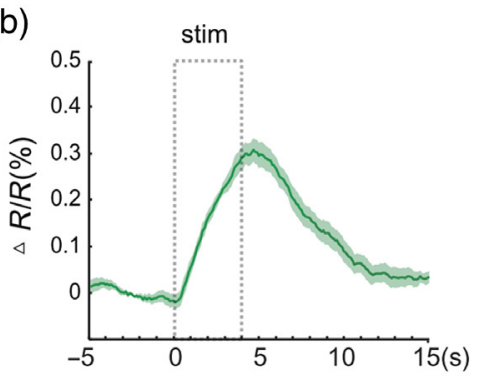

(c)

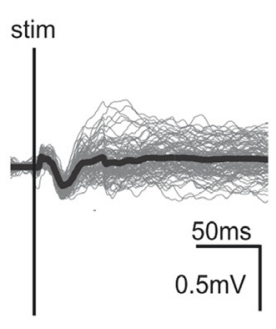

(d)

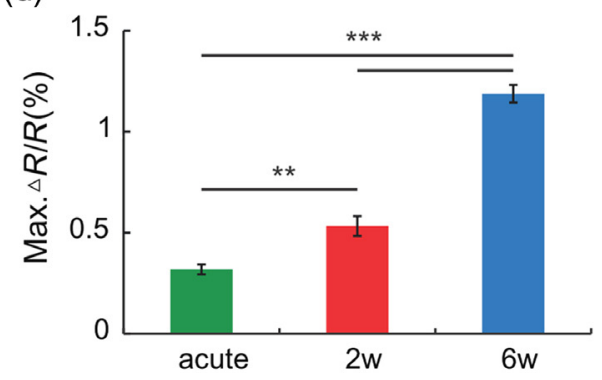

(e)

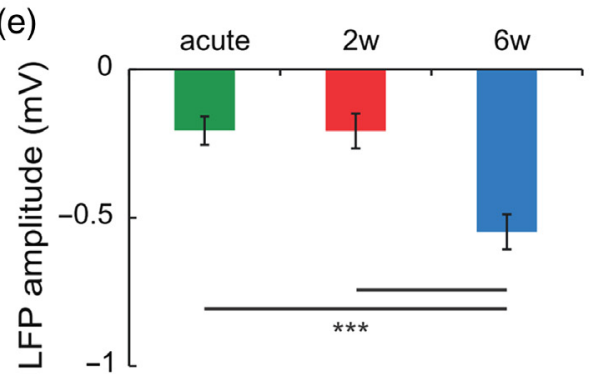

Fig. 12 Hemodynamic change and LFP postsoft window installation. (a) Spatial activation map of ORIS imaging during single whisker stimulation. (b) Graph of $\Delta R / R$ change $(n=7)$. (c) LFP following C2 whisker single deflection (trials $=100$ ). (d) Peak value of $\Delta R / R$ change (acute: $n=7,2$ weeks, 6 weeks: $n=10, p$ value: acute versus 2 weeks $=0.003$, acute versus 6 weeks, 2 weeks versus 6 weeks $=p$ value $>0.001$ ). (e) Peak amplitude of LFP following C2 whisker single deflection (trials $=$ 500 , all $p$ values $<0.001$.

6 weeks, 2 weeks versus 6 weeks $=p$ value $>0.001$; trials $=$ 500 in each group; Figs. 12(c) and 12(e)]. This demonstrated that surgical process can affect hemodynamic response and neuronal activity immediately.

\section{Disclosures}

No conflicts of interests, financial or otherwise, are declared by all authors.

\section{Acknowledgments}

This work was supported by a National Research Foundation of Korea (NRF) grant funded by the Korea government (MSIT) (Grant No. 2017R1A2B4009350) and by Basic Science
Research Program through the National Research Foundation of Korea (NRF) funded by the Ministry of Education (Grant No. 2017R1A6A1A03015642). This work was also supported by the Institute for Basic Science (IBS-R015-D1) in Republic of Korea. We thank Drs. C. Heo and H. Kwak for material supports and Profs. S. G. Kim, M. Choi, J. Choi, and H. Kim for helpful discussion.

\section{References}

1. A. M. Blamire et al., "Interleukin- $1 \beta$-induced changes in blood-brain barrier permeability, apparent diffusion coefficient, and cerebral blood volume in the rat brain: a magnetic resonance study," J. Neurosci. 20(21), 8153-8159 (2000). 
2. A. Holtmaat et al., "Long-term, high-resolution imaging in the mouse neocortex through a chronic cranial window," Nat. Protocols 4(8), 1128-1144 (2009).

3. G. Yang et al., "Thinned-skull cranial window technique for long-term imaging of the cortex in live mice," Nat. Protocols 5(2), 201-208 (2010).

4. P. J. Drew et al., "Chronic optical access through a polished and reinforced thinned skull," Nat. Methods 7(12), 981-984 (2010).

5. A. Y. Shih et al., "A polished and reinforced thinned-skull window for long-term imaging of the mouse brain," J. Vis. Exp. 61, e 3742 (2012).

6. C. J. Roome and B. Kuhn, "Chronic cranial window with access port for repeated cellular manipulations, drug application, and electrophysiology," Front. Cell. Neurosci. 8, 379 (2014).

7. C. Heo et al., "A soft, transparent, freely accessible cranial window for chronic imaging and electrophysiology," Sci. Rep. 6, 27818 (2016).

8. H. Uhlirova et al., "Cell type specificity of neurovascular coupling in cerebral cortex," Elife 5, e14315 (2016).

9. T. L. Roth et al., "Transcranial amelioration of inflammation and cell death after brain injury," Nature 505(7482), 223-228 (2014).

10. H. T. Xu et al., "Choice of cranial window type for in vivo imaging affects dendritic spine turnover in the cortex," Nat. Neurosci. 10(5), 549-551 (2007).

11. D. Guo et al., "In vivo two-photon imaging of astrocytes in GFAP-GFP transgenic mice," PLoS One 12(1), e0170005 (2017).

12. D. X. Hammer et al., "Longitudinal vascular dynamics following cranial window and electrode implantation measured with speckle variance optical coherence angiography," Biomed. Opt. Express 5(8), 2823-2836 (2014).

13. H. Girouard and C. Iadecola, "Neurovascular coupling in the normal brain and in hypertension, stroke, and Alzheimer disease," J. Appl. Physiol. 100(1), 328-335 (2006).

14. E. I. Krook-Magnuson et al., "Tonically active inhibition selectively controls feedforward circuits in mouse barrel cortex," J. Neurophysiol. 100(2), 932-944 (2008).

15. M. Pekny and M. Nilsson, "Astrocyte activation and reactive gliosis," Glia 50(4), 427-434 (2005).

16. F. Aloisi, "Immune function of microglia," Glia 36(2), 165-179 (2001).

17. C. Farina, F. Aloisi, and E. Meinl, "Astrocytes are active players in cerebral innate immunity," Trends Immunol. 28(3), 138-145 (2007).

18. T. Li and S. Zhang, "Microgliosis in the injured brain: infiltrating cells and reactive microglia both play a role," Neuroscientist 22(2), 165-170 (2016).

19. M. Pekny and M. Pekna, "Astrocyte reactivity and reactive astrogliosis: costs and benefits," Physiol. Rev. 94(4), 1077-1098 (2014).

20. T. Tikka et al., "Minocycline, a tetracycline derivative, is neuroprotective against excitotoxicity by inhibiting activation and proliferation of microglia," J. Neurosci. 21(8), 2580-2588 (2001).

21. P. J. Bergold, "Treatment of traumatic brain injury with anti-inflammatory drugs," Exp. Neurol. 275, 367-380 (2016).

22. S. G. A. Baki et al., "Minocycline synergizes with $\mathrm{N}$-acetylcysteine and improves cognition and memory following traumatic brain injury in rats," PLoS One 5(8), e12490 (2010).

23. M. Haber et al., "Minocycline plus $\mathrm{N}$-acetylcysteine synergize to modulate inflammation and prevent cognitive and memory deficits in a rat model of mild traumatic brain injury," Exp. Neurol. 249, 169-177 (2013).

24. S. U. Kim and J. de Vellis, "Microglia in health and disease," J. Neurosci. Res. 81(3), 302-313 (2005).

25. G. W. Kreutzberg, "Microglia: a sensor for pathological events in the CNS," Trends Neurosci. 19(8), 312-318 (1996).

26. A. Karperien, H. Ahammer, and H. Jelinek, "Quantitating the subtleties of microglial morphology with fractal analysis," Front. Cell. Neurosci. 7, 3 (2013).

27. M. L. Block, L. Zecca, and J.-S. Hong, "Microglia-mediated neurotoxicity: uncovering the molecular mechanisms," Nat. Rev. Neurosci. 8(1), 57-69 (2007).

28. K. Riazi et al., "Microglial activation and TNF $\alpha$ production mediate altered CNS excitability following peripheral inflammation," Proc. Natl. Acad. Sci. U. S. A. 105(44), 17151-17156 (2008).
29. E. S. Wohleb, "Neuron-microglia interactions in mental health disorders: 'for better, and for worse'," Front. Immunol. 7, 544 (2016).

30. U.-K. Hanisch and H. Kettenmann, "Microglia: active sensor and versatile effector cells in the normal and pathologic brain," Nat. Neurosci. 10(11), 1387-1394 (2007).

31. A. Familian et al., "Inhibitory effect of minocycline on amyloid $\beta$ fibril formation and human microglial activation," Glia 53(3), 233-240 (2006).

32. T. J. Seabrook et al., "Minocycline affects microglia activation, $\mathrm{A} \beta$ deposition, and behavior in APP-tg mice," Glia 53(7), 776-782 (2006).

33. K. Kobayashi et al., "Minocycline selectively inhibits M1 polarization of microglia," Cell Death Dis. 4, e525 (2013).

34. T. Pang et al., "Minocycline ameliorates LPS-induced inflammation in human monocytes by novel mechanisms including LOX-1, Nur77 and LITAF inhibition," Biochim. Biophys. Acta 1820(4), 503-510 (2012).

35. R. Chen et al., "Adaptive filter by using segment proportionate extended correlation LMS algorithm in the double-talk condition," in Int. Symp. Comput. Sci. Comput. Technol., pp. 716-719 (2008).

36. M. R. Metea and E. A. Newman, "Glial cells dilate and constrict blood vessels: a mechanism of neurovascular coupling," J. Neurosci. 26(11), 2862-2870 (2006).

37. I. Bechmann, I. Galea, and V. H. Perry, "What is the blood-brain barrier (not)?" Trends Immunol. 28(1), 5-11 (2007).

38. M. V. Sofroniew, "Astrocyte barriers to neurotoxic inflammation," Nat. Rev. Neurosci. 16(5), 249-263 (2015).

39. D. Attwell et al., "Glial and neuronal control of brain blood flow," Nature 468(7321), 232-243 (2010).

40. G. C. Petzold and V. N. Murthy, "Role of astrocytes in neurovascular coupling," Neuron 71(5), 782-797 (2011).

41. Y. Dong and E. N. Benveniste, "Immune function of astrocytes," Glia 36(2), 180-190 (2001).

42. A. Buffo, C. Rolando, and S. Ceruti, "Astrocytes in the damaged brain: molecular and cellular insights into their reactive response and healing potential," Biochem. Pharmacol. 79(2), 77-89 (2010).

43. S. Robel et al., "Genetic deletion of cdc42 reveals a crucial role for astrocyte recruitment to the injury site in vitro and in vivo," J. Neurosci. 31(35), 12471-12482 (2011).

44. G. R. John et al., "IL-1-regulated responses in astrocytes: relevance to injury and recovery," Glia 49(2), 161-176 (2005).

45. M. Pekny, U. Wilhelmsson, and M. Pekna, "The dual role of astrocyte activation and reactive gliosis," Neurosci. Lett. 565, 30-38 (2014).

46. A. Dalu et al., "A comparison of the inflammatory response to a polydimethylsiloxane implant in male and female Balb/c mice," Biomaterials 21(19), 1947-1957 (2000).

47. N. Bray et al., "Decreased haemodynamic response and decoupling of cortical gamma-band activity and tissue oxygen perfusion after striatal interleukin-1 injection," J. Neuroinflammation 13(1), 195 (2016).

48. M. Fischer, S. Uchida, and K. Messlinger, "Measurement of meningeal blood vessel diameter in vivo with a plug-in for ImageJ," Microvasc. Res. 80(2), 258-266 (2010).

49. A. Hutt, "The anesthetic propofol shifts the frequency of maximum spectral power in EEG during general anesthesia: analytical insights from a linear model," Front. Comput. Neurosci. 7, 2 (2013).

50. J. A. Vizuete et al., "Graded defragmentation of cortical neuronal firing during recovery of consciousness in rats," Neuroscience 275, 340-351 (2014).

51. K. K. Sellers et al., "Anesthesia differentially modulates spontaneous network dynamics by cortical area and layer," J. Neurophysiol. 110(12), 2739-2751 (2013).

Hyejin Park received her PhD in the Department of Bioscience in Sungkyunkwan University. Her research interests in impacts of neuroinflmmation to neurovascular coupling. She mainly uses intrinsic optical signal imaging and local field potential to observe hemodynamic signal and neural activity.

Nayeon You is an integrated PhD student at the Center for Neuroscience Imaging Research. Her research subject is about epilepsy. She focuses on roles of inhibitory neurons in 4-AP epileptic 
Park et al.: Longitudinal study of hemodynamics and dendritic membrane...

seizure. She investigates vessel diameter change and $\mathrm{Ca}^{2+}$ signal using two-photon microscopy.

Juheon Lee is an integrated $\mathrm{PhD}$ student at the Center for Neuroscience Imaging Research. His primary research is acquiring sub-cortical region hemodynamic information and structure using photoacoustic imaging and ultrasound imaging systems.
Minah Suh is a professor at the Department of Biomedical Engineering in Sungkyunkwan University and participating faculty of Center for Neuroscience Imaging Research. Her research subjects are neurovascular coupling, epilepsy, and chronic stress. She primarily focuses on investigating neurovascular coupling under neurological disorder and providing neurobiological platform for novel convergent technologies. 